\title{
Crack growth by grain boundary cavitation in the transient and extensive creep regimes
}

\author{
T.C. WANG ${ }^{1},{ }^{*}$ C.F. SHIH ${ }^{2}$ and A. NEEDLEMAN ${ }^{2}$ \\ ${ }^{1}$ Institute of Mechanics, Chinese Academy of Sciences, Beijing, People's Republic of China \\ ${ }^{2}$ Division of Engineering, Brown University, Providence, Rhode Island 02912, USA
}

Received 30 April 1990; accepted in revised form 4 December 1990

\begin{abstract}
Crack growth due to cavity growth and coalescence along grain boundaries is analyzed under transient and extensive creep conditions in a compact tension specimen. Account is taken of the finite geometry changes accompanying crack tip blunting. The material is characterized as an elastic-power law creeping solid with an additional contribution to the creep rate arising from a given density of cavitating grain boundary facets. All voids are assumed present from the outset and distributed on a given density of cavitating grain boundary facets. The evolution of the stress fields with crack growth under three load histories is described in some detail for a relatively ductile material. The full-field plane strain finite element calculations show the competing effects of stress relaxation due to constrained creep, diffusion and crack tip blunting, and of stress increase due to the instantaneous elastic response to crack growth. At very high crack growth rates the Hui-Riedel fields dominate the crack tip region. However, the high growth rates are not sustained for any length of time in the compact tension geometry analyzed. The region of dominance of the Hui-Riedel field shrinks rapidly so that the near-tip fields are controlled by the HRR-type field shortly after the onset of crack growth. Crack growth rates under various conditions of loading and spanning the range of times from small scale creep to extensive creep are obtained. We show that there is a strong similarity between crack growth history and the behaviour of the $C(t)$ and $C_{t}$ parameters, so that crack growth rates correlate rather well with $C(t)$ and $C_{t}$. A relatively brittle material is also considered that has a very different near-tip stress field and crack growth history.
\end{abstract}

\section{Introduction}

Progress up to 1987, in various aspects of the mechanics and mechanisms of creep crack growth, is reviewed by Riedel [1]. Some developments within the last three years which are pertinent to our studies are discussed in the review articles by Riedel [2] and Saxena [3]. Broadly speaking, approaches to creep crack growth can be classified into two main categories. One approach to creep crack growth is in terms of a relevant characterizing parameter. This approach assumes that the heavily damaged region is confined to a local zone that is surrounded by an annular region in which the stress and deformation states are well approximated by certain small strain singular crack tip fields. When this small scale damage assumption reasonably describes the actual conditions, the appropriate small strain singular fields provide the relevant characterizing parameter for the near tip state, which may be the elastic stress intensity factor $K_{1}$, the time dependent $C(t)$-integral or its steady state limit $C^{*}$. Under such conditions, the effects of remote load, including the elapsed time since load application, and crack geometry on the near tip state are fully communicated by the characterizing parameter. To borrow the terminology of nonlinear fracture mechanics (see e.g. the review article by Hutchinson [4]) we speak of crack growth under such conditions as $K_{1}, C(t)$ or $C^{*}$-controlled growth. The transferability of laboratory test data requires that the state near the tip of the flawed structure be similar to that which existed near the tip of the specimen during the measurements - and this similarity

\footnotetext{
*Visiting Professor, Brown University, August 1988 through December 1989.
} 
is ensured when an autonomous one-parameter singular field controls both near tip states. Presuming that the mechanism of crack growth does not change, the characterizing parameter approach provides a framework for predicting crack growth rates in structures from measurements on test specimens.

At the other extreme is the damage mechanics approach which, by the nature of its formulation, allows full coupling between damage and deformation irrespective of the scale of damage. In the damage mechanics approach the prediction of crack growth requires the solution of a full boundary value problem in each situation, whether it is for the specimen or for the actual structure, and the damage evolution is characterized by one or more internal variables. Thus fracture mechanics concepts, for example, transferability of crack growth data and similitude notions, are not exploited. Hayhurst et al. $[5,6]$ have undertaken such methods of inquiry within the framework of classical damage mechanics based on Kachanov's damage model [7]. Kachanov's model [7] contains no length scale and damage is characterized by a parameter that has no direct physical interpretation, although some qualitative connection can be made with the cavitated area of the grain boundaries. This particular deficiency of the classical damage equations can be corrected by incorporating a description of the damage mechanism into the constitutive model. As an example, Hutchinson [8] models a creeping solid with a dilute concentration of constrained cavitating grain boundary facets as a power law viscous solid containing a distribution of penny-shaped microcracks. This model has been developed into a micromechanically based creep rupture constitutive relation by Tvergaard $[9,10]$. The grain boundary facets are modelled in a continuous, 'smeared out' manner and failure occurs when the cavities on the facets coalesce to form open microcracks. Tvergaard has used this material model in numerical investigations of creep rupture in notched and smooth tensile bars $[11,55]$ and of crack growth in a center cracked panel [12]. In these studies no attempt was made to examine the structure of the near-tip fields nor to correlate crack growth rates with candidate crack tip parameters.

In our investigations we combine a fracture mechanics approach with the micromechanical modelling of creep crack growth by cavity growth and coalescence along grain boundaries. To this end we use the constitutive model developed by Tvergaard $[9,10]$. The computations are designed so that issues related to the dominance of competing singular fields can be answered unambiguously within a framework that accounts for the competing effects of stress relaxation due to constrained creep, diffusion and crack tip blunting, the stress increase due to the instantaneous elastic response to crack growth and the loss of stress carrying capacity due to progressive damage. We do this through full-field nonlinear plane strain finite element analyses of the compact tension specimen geometry. By proper choice of specimen size, load levels and material parameters we are able to investigate crack growth in both the small scale and extensive creep regimes and for the full range of crack growth rates. The mesh employed in our numerical study is sufficiently refined so that questions regarding the zone of dominance and the form of the dependence of crack growth rates on the relevant load and material parameters can be systematically examined. Such studies can provide some insight into the dependence of crack growth rates on micromechanical failure processes and the effects of coupling between damage and deformation.

Within the framework of the fracture mechanics approach, the effects of crack geometry and size, and load history on the near tip state are fully characterized by the relevant fracture parameter. In other words, crack growth rate data versus $C^{*}$ as obtained in a 
laboratory specimen (presuming that $C^{*}$ is the appropriate load parameter, i.e. the HRR type field dominates over a microstructurally relevant size scale) can be interpreted as a correlation of crack growth rate versus $C(t)$. Then, the crack growth rate versus $C^{*}$ data can be applied to crack growth under small scale as well as extensive creep conditions in a larger specimen or in an actual structure as long as $C(t)$ is the appropriate load parameter and that the same fracture/damage mechanism is operative. We explore this idea by comparing the calculated crack growth rates under extensive creep in this study with the small scale creep results obtained in an earlier study by $\mathrm{Li}$ et al. [13]. In this investigation, we analyze the full range of conditions from small scale to extensive creep, examine growth rates over a much greater range than those in [13], and contrast ductile and brittle type material behavior. The broad range of circumstances considered allows us to assess the range of validity of $C(t)$ and $C_{t}$-controlled growth and of the small-scale damage approximation, which is a prerequisite for the transferability of specimen crack growth data to the structure through the $C(t)$ or $C_{t}$ parameter.

\section{Problem formulation}

\subsection{Field equations}

The finite element analysis is based on a convected coordinate Lagrangian formulation of the field equations with the initial unstressed state taken as reference. All field quantities are considered to be functions of convected coordinates, $x^{i}$, which serve as particle labels, and time $t$. This formulation has been employed extensively in previous finite element analyses, e.g. Needleman [14]. In particular, the formulation used in this study is identical to that in $\mathrm{Li}$, Needleman and Shih [13]. For completeness, the basic equations are given here; further details are in $\mathrm{Li}$ et al. [13]. The position, relative to a fixed Cartesian frame, of a material point in the initial configuration is denoted by $X$. In the current configuration the material point initially at $\boldsymbol{X}$ is at $\overline{\boldsymbol{X}}$. The displacement vector $\boldsymbol{u}$ and the deformation gradient $\boldsymbol{F}$ are defined by

$$
u=\bar{X}-X, \quad F=\frac{\partial \bar{X}}{\partial X}
$$

Base vectors in the reference configuration (unbarred) and in the current configuration (barred) are given by

$$
\begin{aligned}
& \boldsymbol{g}_{i}=\frac{\partial \boldsymbol{X}}{\partial x^{i}}, \quad \overline{\boldsymbol{g}}_{i}=\frac{\partial \overline{\boldsymbol{X}}}{\partial x^{i}}, \\
& \boldsymbol{g}^{i}=g^{i j} \boldsymbol{g}_{j}, \quad \overline{\boldsymbol{g}}^{i}=\bar{g}^{i j} \overline{\boldsymbol{g}}_{j},
\end{aligned}
$$

where $g^{i j}$ and $\bar{g}^{i j}$ are, respectively, the inverses of the metric tensors $g_{i j}=\boldsymbol{g}_{i} \cdot \boldsymbol{g}_{j}$ and $\bar{g}_{i j}=\overline{\boldsymbol{g}}_{\boldsymbol{i}} \cdot \overline{\boldsymbol{g}}_{\boldsymbol{j}}$.

Attention is confined to quasi-static deformations and body forces are presumed absent. At a given stage of the deformation history, the current values of all field quantities, i.e. stresses, 
strains and material properties, are supposed known. The rate boundary value problem is formulated by expanding the principle of virtual work about the current state to obtain

$$
\Delta t \int_{V}\left(\dot{\tau}^{i j} \delta \dot{\eta}_{j i}+\tau^{i k} \dot{u}_{, k}^{j} \delta \dot{u}_{j, i}\right) \mathrm{d} V=\Delta t \int_{S} \dot{T}^{j} \delta \dot{u}_{j} \mathrm{~d} S-\left[\int_{V} \tau^{i k} \delta \dot{\eta}_{k i} \mathrm{~d} V-\int_{S} T^{j} \delta \dot{u}_{j} \mathrm{~d} S\right]
$$

Here, $\tau^{i j}$ are the contravariant components of Kirchhoff stress $(\tau=J \boldsymbol{\sigma}$, where $J=\operatorname{det}(\boldsymbol{F})$ and $\boldsymbol{\sigma}$ is the Cauchy stress) on the deformed convected coordinate net; $V$ and $S$ are the volume and surface, respectively, of the body in the reference configuration; $\Delta t$ is the time step; $(\cdot)=\partial() / \partial t$ at fixed $x^{i}$ and ()$_{, i}$ denotes covariant differentiation in the reference frame. The components $i^{i k}$ are the contravariant components of the convected derivative of the Kirchhoff stress on the deformed base vectors, i.e. the tensor is $i^{i j} \overline{\boldsymbol{g}}_{\boldsymbol{i}} \overline{\boldsymbol{g}}_{\boldsymbol{j}}$. The bracketed term on the right hand side of (2.4) is an equilibrium correction term included to reduce drifting of the solution from the true equilibrium path due to the discrete time step.

The nominal traction components, $T^{i}$, and Lagrangian strain rate components, $\dot{\eta}_{i j}$, are given by

$$
\begin{aligned}
& T^{i}=\left(\tau^{i j}+\tau^{k j} u_{. k}^{i}\right) v_{j}, \\
& \dot{\eta}_{i j}=\frac{1}{2}\left(F_{. i}^{k} \dot{u}_{k, j}+F_{. j}^{k} \dot{u}_{k, i}\right),
\end{aligned}
$$

where $v_{j}$ are the covariant components of the surface normal in the reference configuration.

\subsection{Constitutive relations}

The material is characterized as an elastic-power law creeping material, with an additional contribution to the rate of creep deformation arising from a given density of cavitating grain boundary facets as depicted in Fig. 1. The grain boundary voids grow by a combination of diffusion and creep, with the rate of cavity growth constrained by the rate of dislocation creep in the surrounding grains, Dyson [15], Rice [16]. A full finite deformation phenomenological constitutive description of this process has been developed by Tvergaard $[9,10]$, extending work of Hutchinson [8] and Rice [16].

The rate of deformation tensor, $\boldsymbol{d}$, is the symmetric part of $\dot{\boldsymbol{F}} \cdot \boldsymbol{F}^{-1}$ and is written as the sum of an elastic part, $\boldsymbol{d}^{e}$, and a creep part, $\boldsymbol{d}^{c}$, so that

$$
d=d^{e}+d^{c}
$$

with

$$
\boldsymbol{d}^{e}=\frac{1+v}{E} \hat{\boldsymbol{\sigma}}-\frac{v}{E}(\hat{\boldsymbol{\sigma}}: I) I
$$

Here $\hat{\sigma}$ is the Jaumann rate of Cauchy stress, $\boldsymbol{I}$ is the identity tensor, $E$ is Young's modulus and $v$ is Poisson's ratio. The notation $\boldsymbol{A}: \boldsymbol{B}$ denotes the dyadic product; i.e. $\boldsymbol{A}: \boldsymbol{B}=A^{i j} B_{j i}$. 


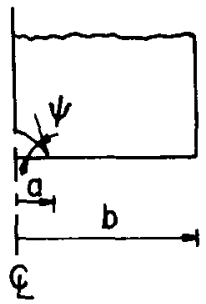

(a)

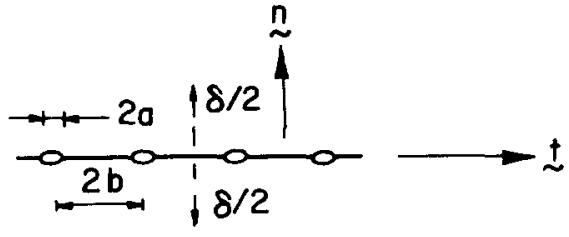

(b)

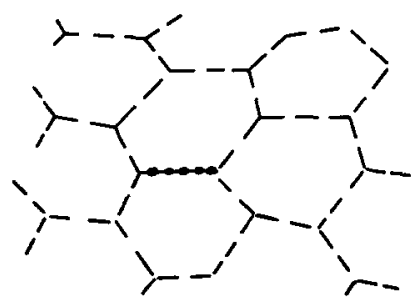

(c)

Fig. 1. (a) Spherical-caps shape of a single cavity. (b) Equally spaced cavities on a grain boundary. (c) An isolated, cavitated grain boundary facet in a polycrystalline material.

The expression for the creep part of the rate of deformation is that derived by Hutchinson [8] for a polycrystalline material undergoing creep-constrained grain boundary cavitation, modified by Tvergaard [9] to account for a non-zero normal stress acting on the cavity facets,

$$
\boldsymbol{d}^{c}=\dot{\varepsilon}_{0}\left(\frac{\bar{\sigma}}{\sigma_{0}}\right)^{n}\left[\frac{3}{2} \frac{\sigma^{\prime}}{\bar{\sigma}}+\rho\left\{\frac{3}{2} \frac{n-1}{n+1} \frac{\sigma^{\prime}}{\bar{\sigma}}\left(\frac{s-\sigma_{n}}{\bar{\sigma}}\right)^{2}+\frac{2}{n+1}\left(\frac{s-\sigma_{n}}{\bar{\sigma}}\right) \bar{n} \otimes \bar{n}\right\}\right],
$$

where $n$ is the creep exponent, $\boldsymbol{x} \otimes \boldsymbol{y}$ denotes the tensor product having components $x^{i} y^{j}$ and

$$
\boldsymbol{\sigma}^{\prime}=\boldsymbol{\sigma}-\sigma_{m} \boldsymbol{I} \quad \sigma_{m}=\frac{1}{3}(\boldsymbol{\sigma}: \boldsymbol{I}) \quad \bar{\sigma}^{2}=\frac{3}{2} \boldsymbol{\sigma}^{\prime}: \boldsymbol{\sigma}^{\prime} \quad s=\overline{\boldsymbol{n}} \cdot \boldsymbol{\sigma} \cdot \overline{\boldsymbol{n}}
$$

Here, $s$ represents the macroscopic normal stress on cavitating facets with normal $\bar{n}$ in the current configuration. The parameters $\sigma_{n}$ and $\rho$ are internal variables; $\sigma_{n}$ is the average normal stress in the vicinity of the voids and $\rho$ is a measure of the density of the cavitating facets, which, in the calculations is a prescribed constant. Evolution equations are specified for $\overline{\boldsymbol{n}}$ and for $\sigma_{n}$. The evolution of $\bar{n}$ is obtained from the geometrical relation

$$
\bar{n}=\frac{n \cdot F^{-1}}{\left|n \cdot F^{-1}\right|}
$$

where || denotes the norm of a vector and $\boldsymbol{n}$ is the normal to the cavitating grain facet in the reference configuration.

The evolution equation for $\sigma_{n}$ comes from the description of grain boundary void growth,

$$
\frac{\dot{V}}{\pi b^{2}}-\frac{V}{\pi b^{2}}\left[\frac{\boldsymbol{t} \cdot \dot{\boldsymbol{\eta}} \cdot \boldsymbol{t}}{\boldsymbol{t}^{T} \cdot \boldsymbol{F}^{T} \cdot \boldsymbol{F} \cdot \boldsymbol{t}}\right]=2 R \beta \frac{s-\sigma_{n}}{\bar{\sigma}} \dot{\varepsilon}_{0}\left(\frac{\bar{\sigma}}{\sigma_{0}}\right)^{n}
$$


where $\boldsymbol{t}$ is a unit vector perpendicular to $\boldsymbol{n}, \dot{\boldsymbol{\eta}}=\dot{\eta}_{i j} \boldsymbol{g}^{i} \boldsymbol{g}^{j}, R$ is the current radius of the circular grain boundary facet, $\beta=(4 / \pi)(1+3 / n)^{-1 / 2}$ and the void volumetric growth rate is written as

$$
\dot{V}=\dot{V}_{1}+\dot{V}_{2}
$$

Here, $\dot{V}_{1}$ is the contribution to the volumetric growth rate due to grain boundary diffusion, and $\dot{V}_{2}$ is the contribution due to dislocation creep in the adjacent grains.

Based on the numerical studies of grain boundary void growth of Needleman and Rice [17] and Sham and Needleman [18], the following approximate expressions for the volumetric growth rate are used,

$$
\begin{aligned}
& \dot{V}_{1}=4 \pi\left(\frac{L^{3} \overline{\bar{\varepsilon}} c}{\bar{\sigma}}\right) \frac{\sigma_{n}-(1-f) \sigma_{s}}{\ln (1 / f)-(3-f)(1-f) / 2}, \\
& \dot{V}_{2}= \begin{cases}2 \pi \dot{\bar{\varepsilon}}^{c} a^{3} h(\varphi)\left[\alpha_{n}+\beta_{n}\right]^{n}\left(\sigma_{m} / \bar{\sigma}\right), & \text { if }\left(\left|\sigma_{m} / \bar{\sigma}\right| \leqslant 1\right) \\
\operatorname{sign}\left(\sigma_{m}\right) 2 \pi \dot{\bar{\varepsilon}}^{c} a^{3} h(\varphi)\left[\alpha_{n}\left|\sigma_{m} / \bar{\sigma}\right|+\beta_{n}\right]^{n}, & \text { if }\left(\left|\sigma_{m} / \bar{\sigma}\right|>1\right)\end{cases}
\end{aligned}
$$

where $\alpha_{n}=\frac{3}{2} n, \beta_{n}=(n-1)(n+0.4319) / n^{2}, \dot{\bar{\varepsilon}}^{c}=\dot{\varepsilon}_{0}\left(\bar{\sigma} / \sigma_{0}\right)^{n}$ and $f$ is defined as $f=\max \left\{a^{2} / b^{2}, a^{2} /\right.$ $\left.(a+1.5 L)^{2}\right\}$, with $a$ and $b$ being the cavity radius and half spacing along the grain boundary, respectively. As in Li et al. [13], the sintering stress $\sigma_{s}$ is taken to be zero.

The function $h(\varphi)$ in (2.14) and (2.15) characterizes the axisymmetric void shape through $V=4 \pi a^{3} h(\varphi) / 3$. For a spherical-caps void shape

$$
h(\varphi)=\left[(1+\cos \varphi)^{-1}-0.5 \cos \varphi\right] / \sin \varphi,
$$

where $\varphi=70^{\circ}$ is a representative value for the angle that the void surface makes with the plane of the grain boundary.

It is assumed that surface diffusion is sufficiently rapid to maintain the quasi-equilibrium, spherical-caps cavity shape so that the cavity size is specified by the single parameter $a$. Creep deformation alone would tend to elongate the voids in the tensile direction, while very rapid grain boundary diffusion would tend to give crack-like cavities. The material length parameter, $L$, is defined as $L=\left(\bar{D} \bar{\sigma} / \dot{\bar{\varepsilon}}^{c}\right)^{1 / 3}$ with $\bar{D}=D \delta \Omega / k T$, where $D \delta$ is the diffusion coefficient on the grain boundary, $\Omega$ is the atomic volume and $k T$ is the energy per atom measure of temperature. The influence of triaxial stressing on grain boundary cavity growth was investigated by Sham and Needleman [18] who suggested the high triaxiality approximation for the volumetric growth rate in (2.15) while the low triaxiality expression in (2.15) was suggested by Tvergaard $[10]$.

Using (2.8) and (2.9) in (2.7) and inverting gives

$$
\hat{\boldsymbol{\sigma}}=\boldsymbol{L}: \boldsymbol{d}^{e}=\boldsymbol{L}: \boldsymbol{d}-\boldsymbol{Q}
$$

where $\boldsymbol{L}$ denotes the tensor of elastic moduli obtained from (2.8), and $\boldsymbol{Q}=\boldsymbol{L}: \boldsymbol{d}^{\mathrm{c}}$.

For use in the principle of virtual work, (2.4), the stress rate-strain rate relation is expressed in terms of the contravariant components of the convected derivative of Kirchhoff stress on the current base vectors. Using standard kinematic transformations the needed rela- 
tion is

$$
\dot{\tau}^{i j}=C^{i j k l} \dot{\eta}_{k l}-J Q^{i j}
$$

with $Q^{i j}=L^{i j k l} \dot{\eta}_{k l}^{c}, \dot{\eta}_{k l}^{c}=\overline{\boldsymbol{g}}_{i} \cdot \boldsymbol{d}^{c} \cdot \overline{\boldsymbol{g}}_{j}, J=\operatorname{det}(\boldsymbol{F})$ and

$$
C^{i j k l}=J L^{i j k l}+\tau^{i j} \bar{g}^{k l}-\frac{1}{2}\left(\bar{g}^{i k} \tau^{j l}+\bar{g}^{j k} \tau^{i l}+\bar{g}^{i l} \tau^{j k}+\bar{g}^{j l} \tau^{i k}\right) .
$$

The current values of the displacement, $\boldsymbol{u}$, and deformation gradient, $\boldsymbol{F}$, are obtained by time integration and the current value of $\bar{n}$ is calculated from (2.11); $\sigma_{n}$ and the cavity volume growth rate $\dot{V}$ are determined from (2.12) to (2.15). Then, $\dot{A}$ and $\dot{b}$ are calculated from

$$
\begin{aligned}
& \dot{A}=\frac{\boldsymbol{t} \cdot \dot{\boldsymbol{\eta}} \cdot \boldsymbol{t}}{\sqrt{\boldsymbol{t}^{T} \cdot \boldsymbol{F}^{T} \cdot \boldsymbol{F} \cdot \boldsymbol{t}}} A_{0}, \\
& \frac{\dot{b}}{b}=\frac{1}{2} \frac{\dot{A}}{A} .
\end{aligned}
$$

In deriving (2.21), the number of voids per unit reference area is presumed constant, i.e. void nucleation is neglected. The spherical-caps void geometry gives the relation between the cavity radius growth rate $\dot{a}$ and the cavity volume growth rate, $\dot{V}$, as

$$
\dot{a}=\dot{V} /\left[4 \pi a^{2} h(\varphi)\right]
$$

In addition to the elastic and creep properties, $v, n$ and $\sigma_{0} / E$ (the parameter $\dot{\varepsilon}_{0}$ serves to set the time scale), values of the initial void radius, $a_{0}$, the initial void spacing, $b_{0}$, the initial grain facet area, $A_{0}=\pi R_{0}^{2}$, the material dependent length, $L$, and the density and orientation of cavitating grain boundary facets, $\rho$ and $\boldsymbol{n}$, respectively, need to be specified.

\subsection{Solution procedure}

Plane strain finite element calculations are carried out using meshes that model the compact specimen, of width $W$ with crack length $l$, shown in Fig. 2 . The symmetry of the specimen and of the loading about the crack line permits modelling to be confined to half of the specimen. The two finite element meshes used in the computations are shown in Figs. 3 and 4. Each mesh consists of quadrilaterals composed of four 'crossed' linear displacement triangular elements. The mesh shown in Fig. 3 has 1500 elements and that shown in Fig. 4 has 1100 elements. In all cases studied, the relative crack size is $l / W=0.5$.

For the mesh in Fig. 3 the initial crack tip is represented by a semi-circular notch. This mesh is well suited for resolving crack tip blunting, but because the element size increases with distance from the initial crack tip, the amount of growth that can be represented accurately is limited. In Fig. 4 the initial crack tip is represented by an abrupt change in the boundary conditions along $x^{2}=0$. This mesh is not as well suited for resolving blunting as is the mesh in Fig. 3. However, the $6 \times 64$ quadrilateral uniform mesh region in front of the initial crack tip permits larger amounts of crack growth to be resolved. 


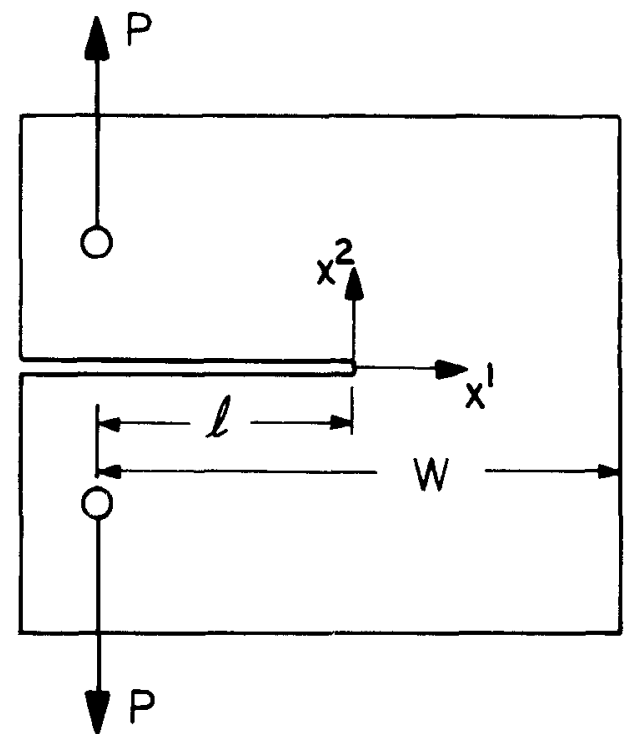

Fig. 2. Compact specimen and definitions.
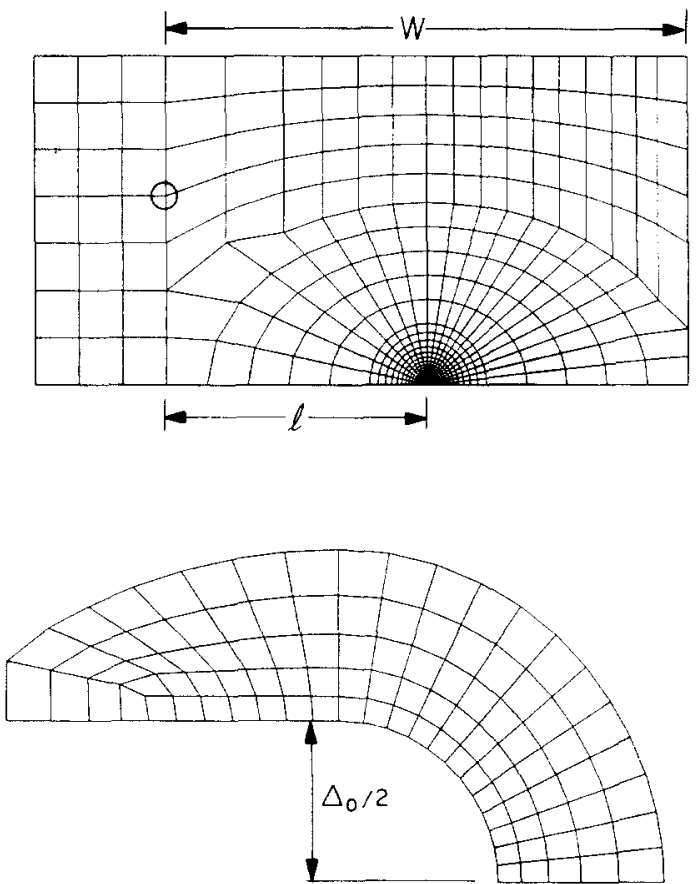

Fig. 3. Finite element mesh for use with small amounts of crack growth, (a) complete mesh, (b) mesh of near-tip region. Each quadrilateral element is composed of four triangular constant strain elements.

In either case, the loading pin is not explicitly modelled and the plane strain specimen is loaded by applying a line force, of magitude $P / B$ per unit thickness, in the $x^{2}$ direction. In a standard compact specimen, the center of the hole containing the loading pin is at $x^{1}=-l, x^{2}=0.275 W$ and the hole radius is $0.125 W$. In the calculations the line force is 

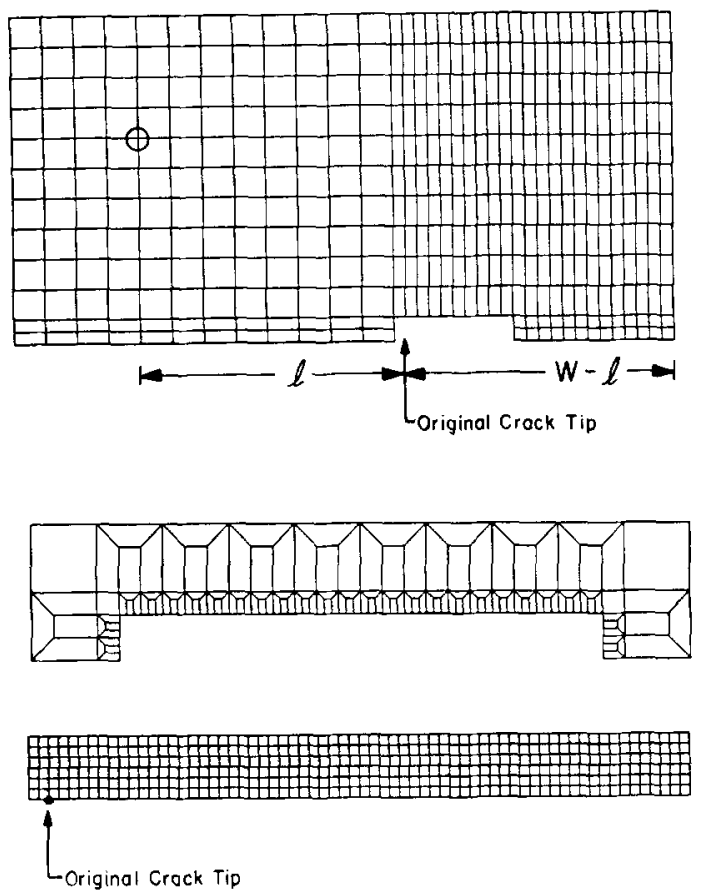

Fig. 4. Finite element mesh for use with large amounts of crack growth, (a) mesh of outer region; (b) mesh of inner region; (c) mesh of crack tip region and path of crack growth. Each quadrilateral element is composed of four triangular constant strain elements.

applied to the node at $x^{1}=-l, x^{2}=0.275 W+0.125 W=0.4 W$. The remaining boundary conditions for the half of the specimen analyzed numerically are symmetry conditions, $T^{1}=0$, $u_{2}=0$, on $x^{2}=0, x^{1}>l$ and traction free conditions $T=0$ on the remainder of the external surface.

The numerical method used in the present calculations is identical to that used by Li et al. [13]. The time integration scheme uses the rate tangent modulus method of Peirce et al. [19] to increase the stable time step. Failure is taken to occur when the cavities on the grain boundary facets coalesce to form open microcracks and then the stresses are reduced to zero using the element vanish technique, Tvergaard $[12,20]$. When the failure criterion is met within an element its stiffness is set to zero and the remaining nodal forces for this element are stepped down to zero over a time interval, $T$, given by

$$
T=\frac{\sigma_{0}}{E \dot{\bar{\varepsilon}}}
$$

where $\dot{\bar{\varepsilon}}$ is the effective strain rate of the failed element at the time when it failed. In (2.23) the strain interval associated with the micro-cracks linking is taken as $\sigma_{0} / E$. For numerical stability reasons, the time increment is adjusted so that at least 15 time steps are used to reduce the nodal forces in an element to zero. In most of the calculations, the criterion for coalescence of grain boundary facets is taken to be that the cavity radius equals the cavity spacing, i.e. $a=b$. However, in order to explore the implications of this criterion for creep crack growth phenomena, some calculations are carried out using $a=0.2 b$ as the failure 
criterion. The parameter $\rho$ in (2.9) is a measure of the density of cavitating grain boundary facets and is related to the number of cavitating facets per unit volume, $\Lambda$, by,

$$
\rho=4 R^{3} \Lambda(n+1)\left(1+\frac{3}{n}\right)^{-1 / 2}
$$

where $n$ is the creep exponent and $R$ is the radius of the penny shaped cracks, Hutchinson $[8]$.

The initial normals $\boldsymbol{n}$ to the cavitating grain boundary facets are taken to coincide with the direction of the maximum principal tensile stress as determined from an elastic finite element solution with $\rho=0$, as in Tvergaard [12] and in $\mathrm{Li}$ et al. [13]. The voids are assumed to be distributed uniformly on the grain boundary facets, which are taken to be circular. It is also assumed that the cavities are crack-like initially so that the initial void volume is zero.

\section{Crack tip fields and characterization parameters}

The central idea of fracture mechanics is this - the singularity that dominates over a microstructurally significant size scale for a given material and load history provides the relevant parameter for correlating crack growth rate data from laboratory specimens and for predicting crack growth and the life of a structure. In a purely viscous material, the near tip stress field is always of the HRR type regardless of whether the crack is stationary or growing and therefore $C^{*}$ is the appropriate load parameter. The stress field of a stationary crack in an elastic-nonlinear viscous material is also of the HRR type though its amplitude, $C(t)$, varies strongly with time in the transient regime. In the extensive creep regime, $C(t) \rightarrow C^{*}$ its steady state value. However, fields of a very different nature evolve near the tip of a growing crack, and therefore questions as to the size of the region of dominance of this field, the HRR-type field as well as the $K$-field (when small scale creep applies), are very important issues.

\subsection{Pertinent results for stationary cracks}

Consider an elastic-nonlinear viscous body to which a sudden load is applied at $t=0$. The instantaneous response of the body is elastic so that the near-tip fields at $t=0$ are given by the well-known elastic singularity

$$
\sigma_{i j}=\frac{K_{\mathrm{I}}}{\sqrt{2 \pi r}} f_{i j}(\theta),
$$

where $r$ and $\theta$ are the polar coordinates centered at the crack tip, $K_{1}$ is the elastic stress intensity factor and $f_{i j}$ are known dimensionless angular functions. If the crack is stationary and the creep exponent $n$ is larger than unity, then creep strain rates dominate over the elastic strain rates and the crack tip fields are of the HRR type. Based on the creep law (2.9) with $\sigma_{n}=0$, Hutchinson [21] has shown that the HRR $[22,23]$ type asymptotic near-tip stresses and strain rates for a 
stationary crack have the form,

$$
\begin{aligned}
& \sigma_{i j}=\sigma_{0}\left[\frac{C(t)}{\dot{\varepsilon}_{0} \sigma_{0} I(n, \rho) r}\right]^{1 /(n+1)} \tilde{\sigma}_{i j}(\theta ; n, \rho), \\
& \dot{\varepsilon}_{i j}^{c}=\dot{\varepsilon}_{0}\left[\frac{C(t)}{\dot{\varepsilon}_{0} \sigma_{0} I(n, \rho) r}\right]^{n /(n+1)} \tilde{\varepsilon}_{i j}(\theta ; n, \rho),
\end{aligned}
$$

where $\tilde{\sigma}_{i j}$ and $\tilde{\varepsilon}_{i j}$ are dimensionless functions of order unity. The dimensionless normalizing factor $I_{n}$ and the $\theta$-variations of $\tilde{\varepsilon}$ depend strongly on $\rho$ while $\tilde{\sigma}$ is only weakly effected, Hutchinson [21]. For constant $\rho$, the amplitude parameter $C(t)$ in (3.2) is given by

$$
C(t)=\int_{\bar{s}}\left[\Phi \bar{n}_{1}-\bar{v} \cdot \sigma \cdot \dot{u}, 1\right] \mathrm{d} \bar{s}
$$

which can be shown to be path independent for all contours $\bar{s}$ around the crack tip in the region dominated by creep strain rates, Bassani and McClintock [24]. In (3.3) $\bar{v}$ is the normal to tha path $\mathrm{d} \bar{s}$ in the current configuration and for a power law creeping solid $\Phi$ is given by

$$
\Phi=\frac{n}{n+1} \sigma^{i j} \dot{\eta}_{i j}
$$

The amplitude factor $C(t)$ depends on the magnitude and history of the applied load, crack length and geometry, material properties and time. If the instantaneously applied load is thereafter held constant, the crack tip stresses relax as the creep strains build up around the tip. At short times a small creep zone surrounds the crack tip - the creep zone being defined as the region where the equivalent creep strain $\bar{\varepsilon}^{c}$ exceeds the equivalent elastic strain $\bar{\varepsilon}^{e}$ (based on deviatoric elastic strains). We denote the characteristic length of the creep zone by $r_{c}$. Well within the creep zone the $C(t)$-integral (3.3) is path-independent (since $\dot{\varepsilon}_{i j}^{c} \gg \dot{\varepsilon}_{i j}^{e}$ ), and it is this value of $C(t)$ which is the amplitude of the HRR-type fields (3.2). For power law creep response, Riedel and Rice [25] and Ohji, Ogura and Kubo [26] have shown that the short time fields are self-similar and that there is an inner zone where the spatial dependence of the stress as well as the creep strain are given by the HRR form. In this regime, they deduced that the relaxation of $C(t)$ is given by

$$
C(t)=\frac{\left(1-v^{2}\right) K_{I}^{2}}{(n+1) E t} \quad \text { for } \quad t>0
$$

The result in (3.5) was shown through full-field calculations to be a good approximation to the actual behavior of $C(t)$ by Bassani and McClintock [24], Ohji et al. [26], Ehlers and Riedel [27] and $\mathrm{Li}$ et al. [28]. The short time field is referred to as the small scale creep field in analogy with small scale yielding in rate-independent elastic-plastic materials. At long times when the creep zone has spread across the entire region, the material behaves like a purely viscous solid. Under these conditions, referred to as extensive creep or steady state conditions, (3.3) is path-independent over the entire body, and $C^{*}$, which is the limit of the $C(t)$-integral as $t \rightarrow \infty$, 
is the creep analog of the Rice's $J$-integral [29], Ohji, Ogura and Kubo [30], Landes and Begley [31] and Nikbin, Webster and Turner [32]. The long time extensive creep case is analogous to the fully plastic state, which has been widely discussed in the literature, so that fully plastic $J$-solutions for the configurations tabulated in the literature are also the $C^{*}$-solutions for the identical configurations. A typical extensive creep solution has the form

$$
C^{*}=\dot{\varepsilon}_{0} \sigma_{0}\left(h(n, \text { geometry })\left(\frac{P}{P_{0}}\right)^{n+1},\right.
$$

where $P_{0}$ is an appropriate reference load, and $h$ is a dimensionless function of $n$ and dimensionless groups of geometric parameters, e.g. Kumar, German and Shih [33]. Under extensive creep, the load line displacement rate $\dot{V}$ is proportional to $l\left(P / P_{0}\right)^{n}$. Combining the latter observation with (3.6) and with a little manipulation we get

$$
C^{*}=\eta \frac{P \dot{V}}{B W},
$$

where $B$ is the specimen thickness, the length $W$ is shown in Fig. 2, and the $\eta$ factor can be determined from the $h_{1}$ and $h_{3}$ fully plastic functions given by Kumar et al. [33]. (The derivation of (3.6) and (3.7) is detailed in Riedel [1] pp. 268-271). The $\eta$ factor is relatively insensitive to the creep exponent, and for a given specimen type it varies only slightly with changes in relative crack size. The $\eta$ factor method is now widely used for the determination of $C^{*}$ from experimental measurements.

The notion of short and long times was made precise by Riedel and Rice [25] and Ohji et al. [26]. To separate the short time from the long time regime, they introduced a transition time, $t_{T}$, given by

$$
t_{T}=\frac{K_{I}^{2}\left(1-v^{2}\right)}{(n+1) E C^{*}}
$$

Thus the transition time depends on the applied load, crack configuration and the elastic and creep properties. For $t \ll t_{T}$ the amplitude of the near-tip fields, $C(t)$, relaxes as $1 / t$ being given by (3.5), while for $t \gg t_{T}$ the amplitude $C(t)$ approaches the steady state value $C^{*}$ of which (3.6) is one representation.

Ehlers and Riedel [27] have shown by full field analysis that the interpolation formula

$$
C(t)=\left[\frac{t_{T}}{t}+1\right] C^{*}
$$

approximates the actual $C(t)$ values fairly accurately for all times. The above result has been confirmed in many subsequent analyses which showed that $C(t)$ is about twice $C^{*}$ at $t=t_{T}$, and that steady state conditions are reached for $t>10 t_{T}$, when $C(t) \approx 1.1 \times C^{*}$, the latter estimate being somewhat geometry dependent, e.g. $[1,28]$.

A major disadvantage of $C(t)$ as a characterizing parameter is that it is not readily related to load point quantities in the transient regime and, therefore, it cannot be directly deter- 
mined by experimental measurements in the transient regime. It must therefore be determined by full-field analysis in which the $C(t)$-integral must be evaluated along contours placed well within the creep zone. If the transition time, $t_{T}$, for the specimen is known, $C(t)$ can be calculated from (3.9) - however, $t_{T}$ depends strongly on creep properties and is not accurately known in general.

These limitations prompted Saxena $[3,34]$ to propose an alternative parameter defined by

$$
C_{t}=-\left.\frac{1}{B} \frac{\partial U_{t}^{*}}{\partial l}\right|_{\dot{V}_{c}},
$$

where $\dot{V}_{c}$ is the load line velocity due to creep, and $U_{t}^{*}=\int P d \dot{V}_{c}$. For a purely nonlinear viscous material with creep exponent $n, U_{i}^{*}=(n /(n+1)) P \dot{V}$ where $P \dot{V}$ is the stress power. In the extensive creep limit, $C_{t}=C^{*}$, but $C(t)$ and $C_{t}$ have different time dependencies in the transient regime. In the small scale creep regime, Bassani, Hawk, and Saxena [35] have related $C_{t}$ to the rate of expansion of the creep zone. Using this result and (3.10), they arrived at the following interpolation formula for all times:

$$
C_{t}=\left[\alpha\left(\frac{t_{T}}{t}\right)^{(n-3) /(n-1)}+1\right] C^{*} .
$$

For large values of $n$, the agreement of the form in (3.11) and (3.9) is easily seen since $\alpha$ is about unity for typical crack geometries. However $C_{t}$ has a significant advantage - it can be computed for all times using direct measurements. It is given by

$$
C_{t}=\frac{P \dot{V}_{c}}{B W}\left(\frac{F^{\prime}}{F}\right)+C^{*}\left(1-\frac{F^{\prime}}{\eta F}\right)
$$

where $F=K_{\mathrm{I}} B \sqrt{W} / P, F^{\prime}=\mathrm{d} F / d(l / W)$. The complete expressions for compact specimens are given in [35]. More recently a slight modification to $C_{t}$ has been proposed by Bassani and Liu [36]. Starting with the observation that in the transient regime $t<t_{T}, C_{t}$ should be proportional to the rate of expansion of the creep zone, $\dot{r}_{c}$, they obtained the result

$$
C_{t}=\frac{P \dot{V}_{c}}{B W}+C^{*}\left(1-\frac{1}{\eta}\right)
$$

The slight difference between (3.12) and (3.13) can be important in some cases. The form in (3.12) does not apply to a constant $K$ specimen, since $F^{\prime}$ vanishes for that specimen, while (3.13) does not involve $F^{\prime}$ and is therefore deemed to be more generally applicable. Saxena, Bassani and their coworkers $[3,34-36]$ have made the case that $C_{t}$ is related to measurable quantities in an experiment in the same way that the $J$-integral is. Nevertheless there is a crucial difference. The $J$-integral is the amplitude of the crack-tip fields of an elastic-plastic material, while $C_{t}$ has this role for a creeping material only under extensive creep conditions. In small scale creep $C_{t}$ is not a direct measure of the instantaneous amplitude of the crack-tip fields but does scale linearly with $\dot{r}_{c}$. The possible characterizing parameter role of $C_{t}$ in the transient crack growth regime is discussed in later sections. 


\subsection{Near-tip fields for growing cracks}

The asymptotic fields around a crack extending in an elastic-nonlinear viscous solid have been derived by Hui and Riedel [37] and verified by full-field analysis by Hui [38]. For a crack growing at a constant or slowly varying velocity in a material with $n>3$, they find that the asymptotic stress and strain fields are of the form,

$$
\begin{aligned}
& \sigma_{i j}=A_{n} \sigma_{0}\left[\frac{\dot{l}(t)}{\dot{\varepsilon}_{0}\left(E / \sigma_{0}\right) r}\right]^{1 /(n-1)} \hat{\sigma}_{i j}(\theta ; n), \\
& \varepsilon_{i j}=A_{n} \frac{\sigma_{0}}{E}\left[\frac{\dot{l}(t)}{\dot{\varepsilon}_{0}\left(E / \sigma_{0}\right) r}\right]^{1 /(n-1)} \hat{\varepsilon}_{i j}(\theta ; n),
\end{aligned}
$$

where $A_{n}$ is a numerical factor depending on $n$, and $\hat{\sigma}_{i j}$ and $\hat{\varepsilon}_{i j}$ are dimensionless functions of order unity. The asymptotic field has an unusual character. The amplitude is uniquely given by the current crack growth rate, $\dot{l}(t)$, so that any growth criterion that involves only combinations of asymptotic stress and strain quantities will lead to a crack growth rate that cannot be related to the applied load. This apparent paradox can be explained by noting that a characteristic length $x_{c}$ must enter into any fracture criterion. Now if $x_{c}$ (the fracture process zone size) is larger than the zone of dominance of the HR singularity, the applied load and crack geometry controls $i$ through the fields that surround the region dominated by the HR singularity. Alternatively, the growth rate can depend on the remote load through a history dependent crack growth mechanism like grain boundary cavitation which was discussed in Section 2.

\subsection{Near-tip fields under non-steady-state conditions}

For the purpose of developing the discussions to follow we designate the characteristic radius of the HR, HRR-type and $K$ fields, and the relevant characteristic dimension of the crack geometry by $r_{\mathrm{HR}}, r_{\mathrm{HRR}}$ and $r_{K}$, and $L$ respectively. To fix ideas, we consider for the moment the crack to be growing under small scale creep conditions. At short times and assuming that the crack has not grown, the crack tip is dominated by the HRR-type field which in turn is surrounded by the $K$-field, i.e. $L>r_{K}>r_{\mathrm{HRR}}>0$. We now suppose the crack begins to grow within a creep zone which is small compared to $L$. Very shortly into the growth, all three singularities coexist, i.e. the HR field is embedded in the HRR-type field which in turn is surrounded by the $K$-field, as found previously by Hawk and Bassani [56]. If the crack growth rate $l$ is smaller than the rate of expansion of the creep zone $\dot{r}_{c}$, then all three singularities continue to coexist. If $\dot{l}$ is faster than $\dot{r}_{c}$, the annular zone dominated by the HRR-type field shrinks and eventually vanishes so that the HR singularity is surrounded by the $K$-field i.e. $L>r_{K}>r_{\mathrm{HR}}>0$. On the other hand if the crack only begins to grow in the extensive creep regime, the $\mathrm{HR}$ field is embedded in the HRR-type field, i.e. $L>r_{\mathrm{HRR}}>r_{\mathrm{HR}}>0$.

Now suppose that the mechanism of fracture is grain boundary cavitation so that a relevant microstructural length is the radius of the grain boundary facet $R_{0}$. This means that the zone of dominance of the HR-field must be larger than $R_{0}$ before we can argue that the HR-field controls the crack growth rate. In other words the question as to which singularity controls the fracture process can be addressed only in conjunction with a characteristic material dimension 
say $x_{c}$. For example, if $x_{c}$ is larger than $r_{\mathrm{HR}}$ but smaller than $r_{\mathrm{HRR}}$, the HRR singularity controls the fracture process and therefore $C(t)$ is the appropriate parameter for correlating crack growth rates. However, suppose that the material is sufficiently brittle and the crack growth rate is sufficiently fast so that creep zone does not extend over a microstructurally significant distance ahead of the crack tip. Then the damage develops in the $K$-controlled region and the elastic stress intensity factor $K_{\mathrm{I}}$ is the appropriate correlation parameter. Finite deformation effects extend over distances comparable to several crack tip openings and present an added complexity.

The above issues have been addressed to varying degrees of satisfaction by Riedel, Bassani, Hui and their coworkers and others [39-46]. In a typical analysis, the crack grows at a rate so as to maintain a critical strain $\varepsilon_{c}$ at a distance $x_{c}$ ahead of the current tip, or alternatively the growth is controlled by grain boundary cavitation. Riedel $[1,2,40]$ has reviewed one-dimensional models for transient crack growth controlled by grain boundary cavitation. In the early studies, the cavities are driven by HRR fields which are undisturbed by cavity growth. Such modelling led to the general result that the crack growth rate is proportional to $\left(C^{*}\right)^{n /(n+1)}$. In later studies, e.g. those based on Kachanov's damage model, the effect of damage on the stress distribution is accounted for [40]. These latter one-dimensional models show that the crack growth rates are not affected in any significant way. The most recent and complete study on the subject is that by Bassani, Hawk and Wu [42]. They carried out full-field finite element plane strain mode I calculations (under small strain assumptions) in which the crack grows according to a critical strain criterion. Their analyses showed the variation of the size of the HR, HRR or $K$ regions with time, with far field load and with crack growth rate. The calculated fields are consistent with estimates, by a matching procedure based on the effective stress, for the size of the HR, HRR, and $K$ dominated regions, as discussed previously by Hawk and Bassani [56].

\section{Numerical results}

\subsection{Near-tip fields}

The material properties are specified by $\sigma_{0} / E=1 / 500, v=0.3$ and $n=5$, and the material parameter $\dot{\varepsilon}_{0}$ serves to set a characteristic time scale, $1 / \dot{\varepsilon}_{0}$. The voids are assumed to be distributed uniformly on the grain boundary facets, which are taken to be circular. The density of cavitating grain boundary facets, $\rho$ in (2.24), is taken to have the constant value 0.2 . The initial values of the cavity radius, the cavity spacing and the radius of the grain boundary facets are related by $a_{0} / b_{0}=0.1$ and $b_{0} / R_{0}=0.1$. Unless specifically stated otherwise, the diffusion constants are specified so that the material length $L$ in (2.14), when calculated from the average normal stress on the cross section $x^{2}=0$ at $t=0$, is $40 a_{0}$ and the onset of failure due to the coalescence of cavities along the grain boundary facet occurs when $a=b$.

For a compact tension specimen of thickness $B$ and $l / W=0.5$, the elastic stress intensity factor, $K_{1}$, is related to the applied load $P$ by, Tada et al. [48],

$$
K_{\mathrm{I}}=13.58 \frac{P \sqrt{l}}{B W}
$$


The initial notch opening $\Delta_{0}$ is indicated in Fig. 3 and, here as in [13], we take $\Delta_{0}=20 R_{0}$. Relative to the specimen, the initial notch tip is relatively sharp since $\Delta_{0} / W=10^{-4}$. The mesh shown in Fig. 3 is suited for accurate resolution of the finite deformation fields during the process of growth initiation and the evolution of the fields for small amounts of crack growth. On the other hand the mesh shown in Fig. 4 is particularly suited for studying extended amounts of crack growth. Thus the complete crack growth behavior is obtained by combining the results obtained from calculations using the meshes in Figs. 3 and 4 . In Fig. 4 the ratio $R_{0} / W$ is $0.2 \times 10^{-4}$ so that if $R_{0}=1.0 \mu \mathrm{m}\left(1 \mu \mathrm{m}=10^{-6} \mathrm{~m}\right)$, then $W=50 \mathrm{~mm}$. In this case the refined mesh zone extends a distance of $6.4 \mathrm{~mm}$ ahead of the initial crack tip. Also the corresponding values of the initial void radius, $a_{0}$, and of the initial void spacing, $b_{0}$, are $0.01 \mu \mathrm{m}$ and $0.1 \mu \mathrm{m}$, respectively.

For the material and geometric parameters given above, we consider three different load levels. The load is applied instantaneously at $t=0$ and is thereafter held constant. The three magnitudes of the load per unit thickness, $P / B$, are chosen so as to correspond to $\left(K_{\mathrm{I}} / \sigma_{0}\right)^{2} / R_{0}=0.625 \times 10^{4}, 2.5 \times 10^{4}$ and $10 \times 10^{4}$, respectively. $K_{\mathrm{I}}$ is computed according to (4.1) and $R_{0}$ is the initial radius of the grain boundary facet. Alternately the load magnitudes correspond to $\left(K_{\mathrm{I}} / \sigma_{0}\right)^{2} / \Delta_{0}=312.5,1250$ and 5000 respectively. The load intensities can also be stated in terms of an effective stress at the distance $R_{0}$ ahead of the crack tip at the instant of load application, at which time the near-tip fields are the elastic fields. Thus, the three cases considered correspond to $\bar{\sigma} / E=0.025,0.05$ and 0.1 at distance $x^{1}=R_{0}$ ahead of the initial crack tip at $t=0$. Li et al. [13] considered two load cases, which were called the slowly growing crack and the fast growing crack cases. To make contact with their boundary layer, plane strain, small scale creep results, our low and high load cases produce short time creep deformation and damage evolution histories in the compact specimen which are similar to those obtained by $\mathrm{Li}$ et al. [13]. Indeed, the load magnitudes $\left(K_{1} / \sigma_{0}\right)^{2} / \Delta_{0}=312.5$ and 1250 are precisely those employed by Li et al. [13], so that direct comparisons between their small scale creep results and the full specimen results obtained in this study can be made.

In all three load cases, crack growth is said to have initiated at the time of the failure of the first element in the mesh shown in Fig. 3. The time to initiation is denoted by $t_{l}$. The transition time $t_{T}$, which has a central role in our analysis, is based on the initial crack length and the applied load. We found that the time to initiation is greatly reduced as the applied load is increased. The times to crack initiation for the low, high and ultra high load cases are $t_{I}=0.188 \times 10^{-2}\left(1 / \dot{\varepsilon}_{0}\right), t_{I}=0.201 \times 10^{-4}\left(1 / \dot{\varepsilon}_{0}\right)$, and $t_{I}=0.294 \times 10^{-5}\left(1 / \dot{\varepsilon}_{0}\right)$, respectively. Li et al. [13] reported values of $0.173 \times 10^{-2}\left(1 / \dot{\varepsilon}_{0}\right)$ and $0.173 \times 10^{-4}\left(1 / \dot{\varepsilon}_{0}\right)$ for the low and high load cases respectively. The good agreement between the initiation times for the similarly loaded near crack tip regions is evidence that the short time creep deformation and damage evolution histories in the compact specimen are very similar to those obtained in [13].

The near-tip fields prior to crack growth are nearly identical to those discussed by Li et al. [13] so that only the main features of the field are noted. In all cases, the full-field finite element solutions within the region $2 \Delta<r<r_{c} / 5$ agreed well with the small strain HRR field (3.2); $\Delta$ is the current opening of the tip and its precise definition is given below. In the crack-tip region $r<2 \Delta$ finite deformation effects are significant and the details of these fields for the cavitating material considered here and for a creeping material in the absence of damage, have been 
discussed by $\mathrm{Li}$ et al. $[13,28]$. Our calculations for stationary cracks did confirm the validity of the body of results in Section 3.1. $C(t)$ did relax as $(1 / t)$ while $C_{t}$ starts at a lower value and decays more gradually so that $C(t)>C_{t}$ for short times. For $t>t_{T}, C_{t} \approx C(t)$.

The results for the load case corresponding to $(\bar{\sigma} / E)_{t=0}=0.025$ or $\left(K_{1} / \sigma_{0}\right)^{2} / \Delta_{0}=312.5$, is discussed first. We note that $\bar{\sigma} / E=0.025$ is the effective stress of the elastic fields at distance $x^{1}=R_{0}$ ahead of the initial crack tip at $t=0$. The transition time for the specimen is $0.145 \times 10\left(1 / \dot{\varepsilon}_{0}\right)$, so that $t_{I} / t_{T}=1.30 \times 10^{-3}$. This load case was called the slowly growing crack case by $\mathrm{Li}$ et al. [13] because the calculated crack growth rate in their analysis is less than the growth rate of the creep zone. The good agreement between the near-tip fields of the growing crack (calculated along the first row of elements, $\theta \approx 0$ ) and the HRR fields can be seen in Figs. 5 and 6. The fields plotted in Fig. 5 were obtained using the mesh shown in Fig. 3 and pertain to small amounts of crack growth and for times smaller than the transition time. Finite deformation effects at the early stages of growth can be seen in Fig. 5a. The stress distributions in Fig. 5 are very similar to those obtained in [13] for the slowly growing crack. Figure 6 shows the fields for times beyond the transition time and for larger amounts of growth - they were obtained using the mesh in Fig. 4. For these

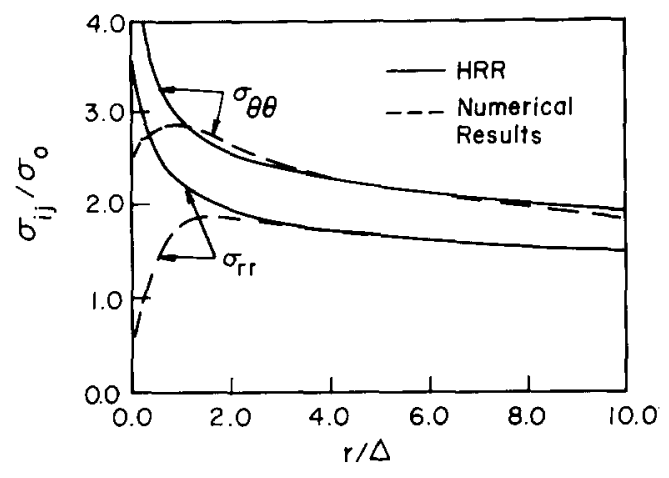

(a)

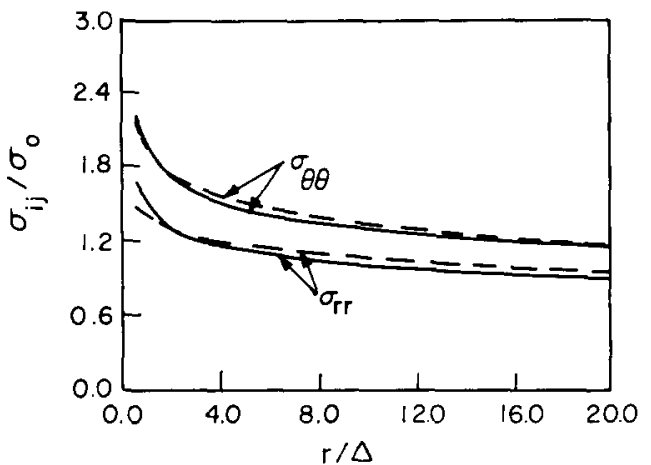

(b)

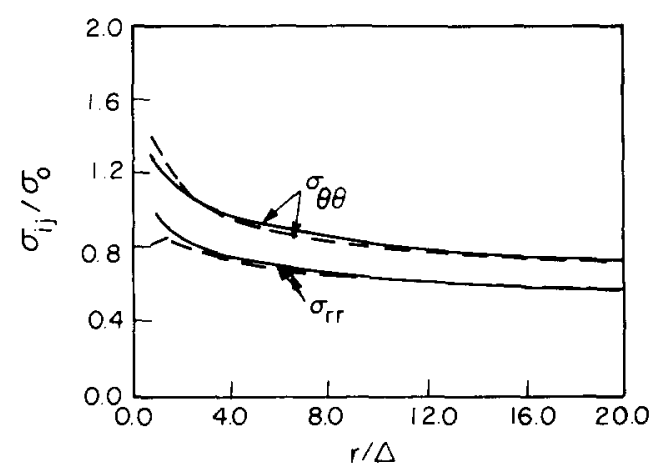

(c)

Fig. 5. Low load case, $(\bar{\sigma} / E)_{t=0}=0.025$. Comparison of the computed stress fields with the HRR type singular field at $t / t_{T}=0.00157,0.0119,0.239$ and for crack growths $\left(l-l_{0}\right) /\left(W-l_{0}\right)=0.555 \times 10^{-4}, 0.668 \times 10^{-3}, 0.135 \times 10^{-2}$ respectively. The mesh in Fig. 3 is used for the calculation. Distance is normalized by the current opening at the initial notch tip. 


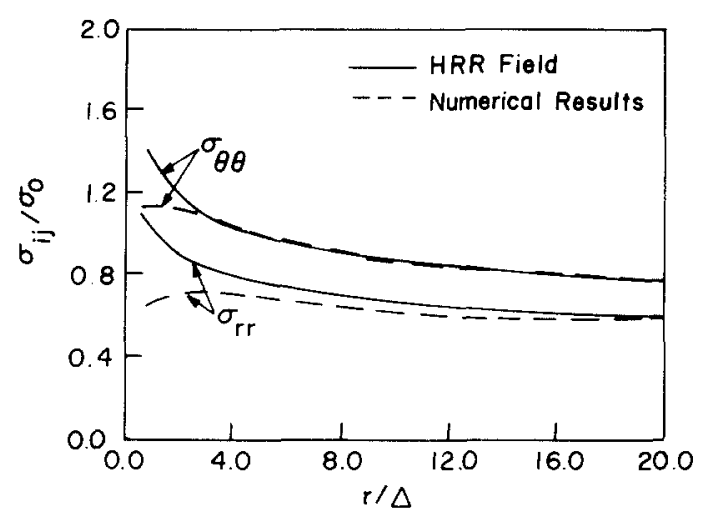

(a)

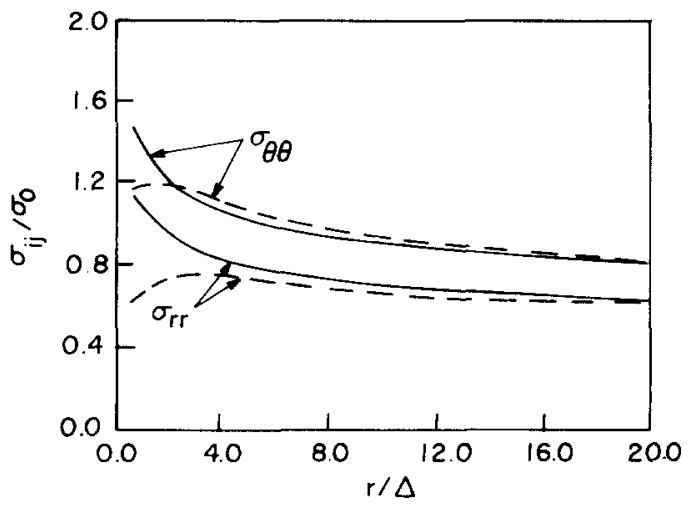

(b)

Fig. 6. Low load case, $(\bar{\sigma} / E)_{t=0}=0.025$. Comparison of the computed stress fields with the HRR type singular fields at $t / t_{T}=12.76,33.62$, and for crack growths $\left(l-l_{0}\right) /\left(W-l_{0}\right)=0.013,0.0475$ respectively. The mesh in Fig. 4 is used for the calculation. Distance is normalized by $\Delta$ defined as $J / \sigma_{0}$ where $J$ is the current value.

times, the specimen is in the extensive creep regime and the stress distribution is essentially unchanging.

In Fig. 5, as for all calculations based on the mesh in Fig. 3, $\Delta$ is the current opening at the initial notch tip, i.e. $\Delta$ is always measured at the node at which $\Delta_{0}$ is defined (see Fig. 3 ). For calculations based on the mesh in Fig. 4, the current opening $\Delta$ is defined by $J / \sigma_{0}$, where $J$ is calculated from its finite strain line integral expression, Rice [29], Eshelby [49], using current values of field quantities. This definition of $\Delta$ is for the sole purpose of providing a normalization length so as to allow us to piece together results obtained from the meshes in Figs. 3 and 4. The essential features of the results are not affected by the different definitions of $\Delta$ that were adopted for the meshes in Figs. 3 and 4. In Figs. 5 and 6, and in subsequent plots, distances are always measured from the current crack tip and defined in the current configuration. For example, the calculated Cartesian components of the Cauchy stress at the current distance of a material point from the current crack tip, are plotted in Figs. 5 through 8 .

For calculations with either mesh, the value of $J$ used in our normalization is evaluated in the remote region where the $J$-integral is practically path-independent. In the transient regime, the $C$-integral is only defined in the region well within the creep zone where creep strain rates dominate. The values of the $C$-integral reported in this study are evaluated along a contour consisting of an annular strip of elements which has a mean radial distance of three elements ahead of the current crack tip. Within the zone dominated by creep strain rates, our calculations do show that the $C$-integral is path-independent. We also calculated $C_{t}$ using (3.12), and for $t>t_{T}$ the value of $C_{t}$ is within 20 percent of $C(t)$ obtained by integrating along contours well within the creep zone. Both the $J$ and $C$ integrals are calculated by the domain integral method of $[50]$.

The high load case, $(\bar{\sigma} / E)_{t=0}=0.05$ or $\left(K_{1} / \sigma_{0}\right)^{2} / \Delta_{0}=1250$, is considered next. This load state was termed the 'fast growing crack' by Li et al. [13] since the calculated crack growth rates in their analysis is faster than the rate of expansion of the creep zone. As in [13], we fix the ratio of diffusion length, $L$, to initial cavity radius, $a_{0}$, at 40 . Since $L$ is stress dependent through $\bar{L}=$ $\left(\bar{D} \bar{\sigma} / \dot{\bar{\varepsilon}}^{c}\right)^{1 / 3}$, a fixed value of $L / a_{0}$ implies a different value of the grain boundary diffusion 


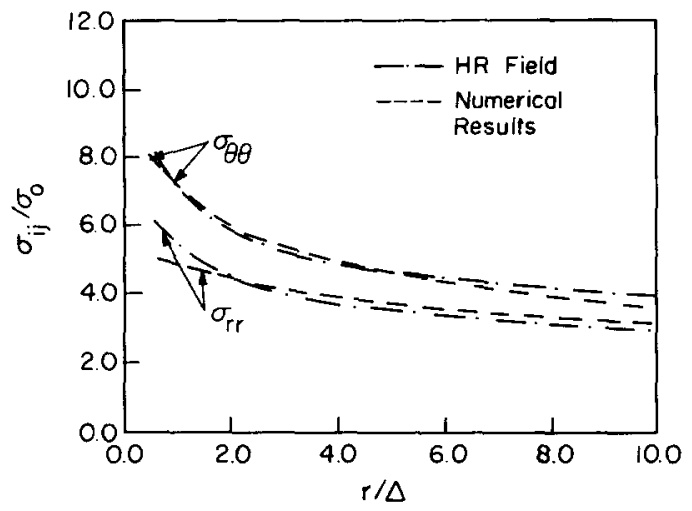

(a)

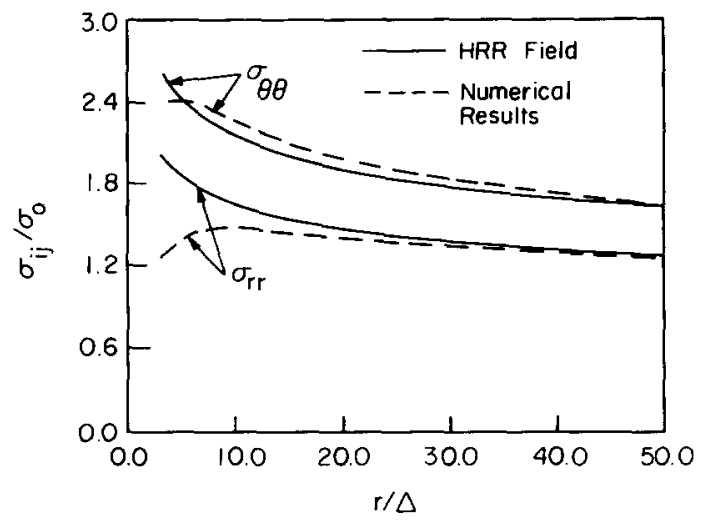

(b)

Fig. 7. High load case, $(\bar{\sigma} / E)_{t=0}=0.05$. (a) Comparison of computed fields with the Hui-Riedel singular fields at $t / t_{T}=0.702 \times 10^{-3}$ and $\left(l-l_{0}\right) /\left(W-l_{0}\right)=0.952 \times 10^{-3}$. The mesh in Fig. 3 is used for the calculation. Distance is normalized by the current opening at the initial notch tip. (b) Comparison of computed fields with the HRR type singular fields at $t / t_{T}=8.38$ and $\left(l-l_{0}\right) /\left(W-l_{0}\right)=0.807 \times 10^{-2}$. The mesh in Fig. 4 is used for the calculation. Distance is normalized by $\Delta$ defined as $J / \sigma_{0}$ where $J$ is the current value.

parameter $\bar{D}$. The time to crack initiation is $0.201 \times 10^{-4}\left(1 / \dot{\varepsilon}_{0}\right)$ which is about one percent of the crack growth initiation time of the preceding low load case. For this load, $t_{T}=0.905 \times$ $10^{-1}\left(1 / \dot{\varepsilon}_{0}\right)$ so that $t_{I} / t_{T}=2.22 \times 10^{-4}$.

Figure $7 \mathrm{a}$ shows the finite element stress fields and the Hui-Riedel [37] fields at $t / t_{T}=$ $0.702 \times 10^{-3}$ and for a small amount of growth. The Hui-Riedel field values are calculated by substituting the numerically determined crack growth rate into (3.14). Good agreement over the spatial range $2.0<r / \Delta<10.0$ can be seen. For times greater than the transition time $t_{T}$, the creep zone ahead of the growing crack tip is well established and, as shown in Fig. $7 \mathrm{~b}$ the near tip stress distribution shifts to the HRR type. The calculated stress fields agreed very well with the HRR field over distances $10<r / \Delta<50$, with $\Delta=J / \sigma_{0}$. Within $r / \Delta<10$, the calculated stress fields deviate from both the HR and HRR fields. It is apparent that the competing effects of crack growth and creep strain rate within an inner region are not amenable to a description by either of these singular fields.

The stress fields for the ultra-high load case, $(\bar{\sigma} / E)_{t=0}=0.1$ or $\left(K_{1} / \sigma_{0}\right)^{2} / \Delta_{0}=5000$, are shown in Fig. 8. The crack growth initiation time is $0.294 \times 10^{-5}\left(1 / \dot{\varepsilon}_{0}\right)$ which is about $1 / 10$ the initiation time for the preceding case. The transition time is $0.566 \times 10^{-2}\left(1 / \dot{\varepsilon}_{0}\right)$, so that $t_{I} / t_{T}=5.19 \times 10^{-4}$. At crack initiation the creep zone is confined to a distance $r \approx 20 R_{0}$ so that the crack tip field for small amounts of growth agreed rather well with the elastic $K_{I}$ field as can be seen in Fig. 8a. For times approaching extensive creep regime, the near-tip fields are more nearly of the HRR type as shown in Fig. $8 \mathrm{~b}$.

\subsection{Crack growth characterization}

One aim of this study is to relate calculated crack growth rates, based on a constitutive relation incorporating a model of cavity growth and coalescence on grain boundaries, to load or crack tip parameters provided by the phenomenological descriptions of creep crack growth. Figures 9,10 and 11 show plots of the time variation of several phenomenological characterizing 


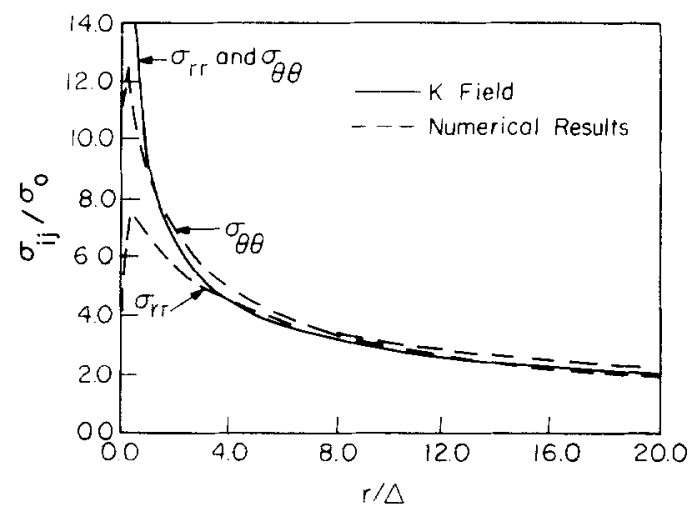

(a)

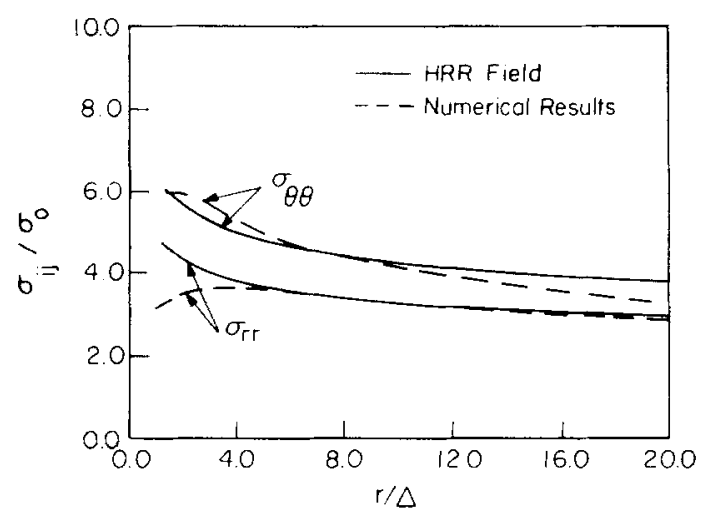

(b)

Fig. 8. Ultra-high load case. $(\bar{\sigma} / E)_{t=0}=0.1$. (a) Comparison of computed fields with the elastic singular fields at $t / t_{T}=0.135 \times 10^{-2}$ and $\left(l-l_{0}\right)\left(W-l_{0}\right)=0.144 \times 10^{-3}$. The mcsh in Fig. 3 is used for the calculation. Distance is normalized by the current opening at the initial notch tip. (b) Comparison of computed fields with the HRR type singular fields at $t_{t}=4.23$ and $\left(l-l_{0}\right) /\left(W-l_{0}\right)=0.796 \times 10^{-1}$. The mesh in Fig. 4 is used for the calculation. Distance is normalized by $\Delta$ defined as $J / \sigma_{0}$ where $J$ is the current value.

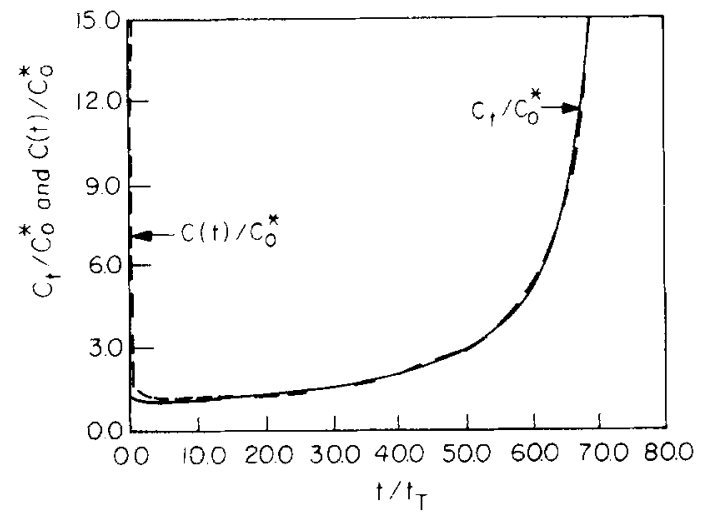

(a)

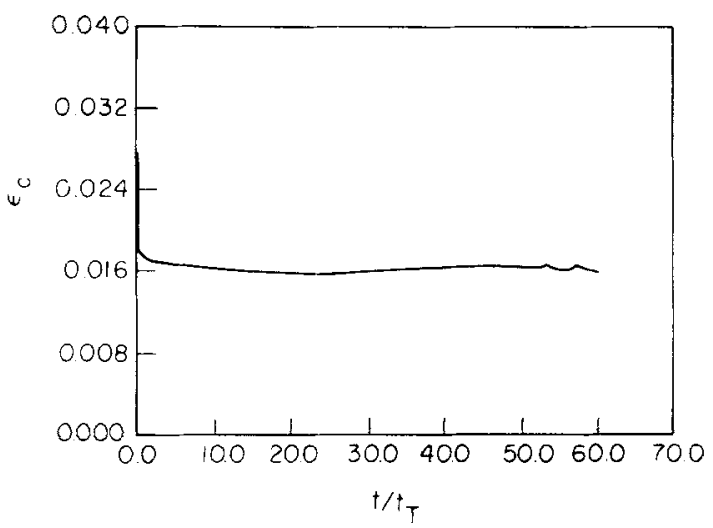

(b)

Fig. 9. Low load case, $(\bar{\sigma}, E)_{t=0}=0.025$. (a) Comparison of $C(t)$ and $C_{t}$. (b) Equivalent creep strain, $\varepsilon_{c}$, at a fixed distance of $50 R_{0}$ ahead of the current crack tip versus time.

parameters for the loading cases, $(\bar{\sigma} / E)_{t}=0=0.025,0.05$ and 0.1 respectively. In each of these figures, (a) shows the time variation of the $C$-integral and of Saxena's $C_{t}$ parameter [34] and (b) shows the average equivalent creep strain at a fixed distance of $50 R_{0}$ directly ahead of the current crack tip. For $R_{0}=1.0 \mu \mathrm{m}$ the equivalent creep strain, $\varepsilon_{c}$, is calculated at the distance of $50 \mu \mathrm{m}$ ahead of the current crack tip. The $C$-integral is calculated using (3.3) and $C_{\text {, }}$ is calculated using (3.12) and the current crack length. In the figures both $C(t)$ and $C_{t}$ are normalized by $C_{0}^{*}$ which is the steady state value of the $C$-integral based on the initial crack length and the appropriate value of the applied load.

Figure 9a shows the behavior of $C(t)$ and $C_{t}$ for the low load case. At short times, $t<t_{T}$, $C(t)>C_{t}$. At intermediate times $C(t) \approx C_{t}$, and for $t \geqslant 3 t_{T}, C_{t}$ and the $C$-integral are nearly equal as they must be since the two parameters are equivalent at extensive creep which is 


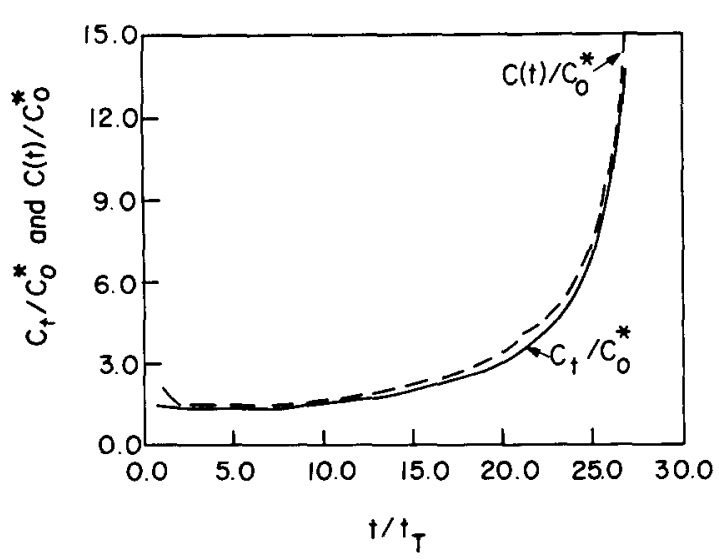

(0)

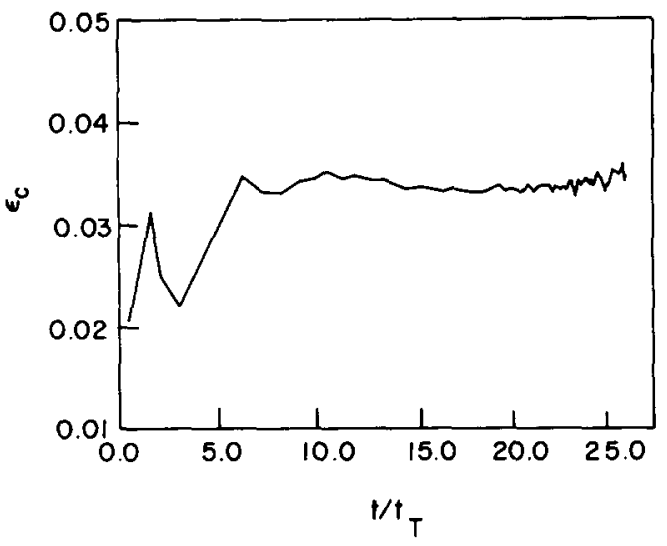

(b)

Fig. 10. High load case, $(\bar{\sigma} / E)_{t=0}=0.05$. (a) Comparison of $C(t)$ and $C_{i}$. (b) Equivalent creep strain, $\varepsilon_{c}$, at a fixed distance of $50 R_{0}$ ahead of the current crack tip versus time.

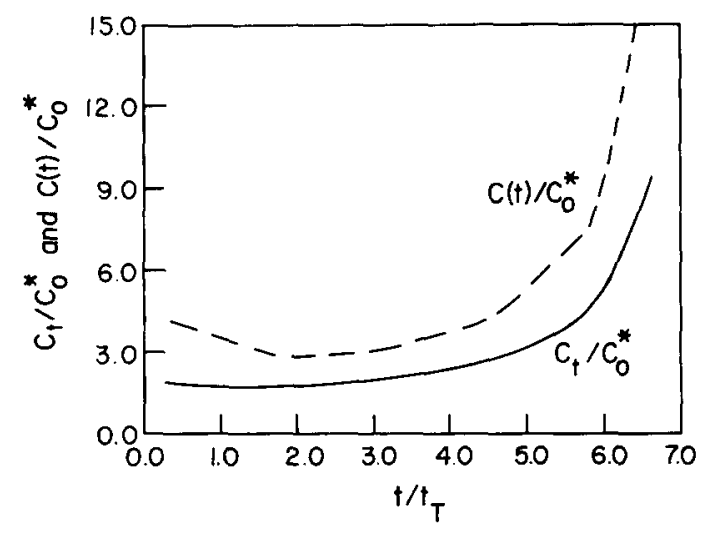

(a)

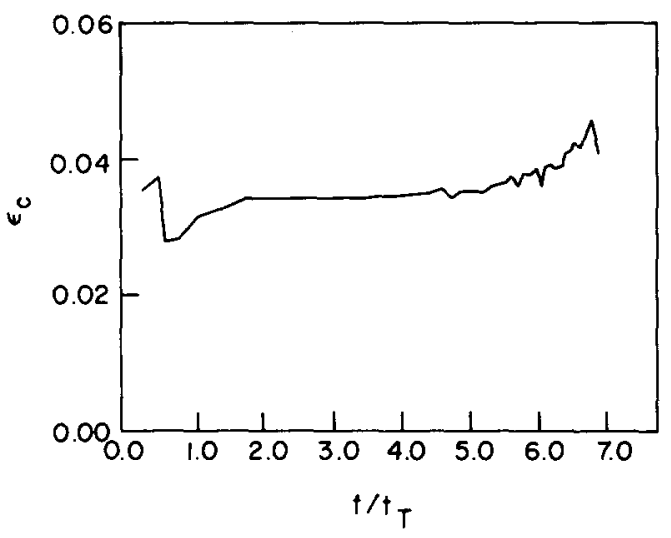

(b)

Fig. 11. Ultra-high load case, $(\bar{\sigma} / E)_{t=0}=0.1$. (a) Comparison of $C(t)$ and $C_{t}$. (b) Equivalent creep strain, $\varepsilon_{t}$, at a fixed distance of $50 R_{0}$ ahead of the current crack tip versus time.

attained when $t$ is greater than about $10 t_{T}$. Figure $9 \mathrm{~b}$ shows the equivalent creep strain, $\varepsilon_{c}$, leveling off at 0.016 for $t>t_{T}$. This suggests that beyond a short transient period (during which $C(t)$ is rapidly decreasing) a critical strain at a critical distance is a suitable growth criterion. Looking ahead to Fig. 12, we note that the time variation of the crack growth rates is quite similar to the time variation of $C(t)$ and $C_{t}$ so that the growth rates appear to correlate with these macroscopic parameters. The behavior of $C_{t}$ and the $C$-integral for the high load case is shown in Fig. 10a. In Fig. 10b, the apparent critical failure strain becomes nearly constant for times greater than about $7 t_{T}$. However, the value of $\varepsilon_{c}$ in Fig. $10 \mathrm{~b}$ is about twice that in Fig. $9 b$.

Figure 11 pertains to the ultra-high load case. Due to the high stresses all the growth that can be accommodated by the mesh occurs over a time period that is only several times longer than the transition time. Growth effects are strong so that the disagreement between $C_{t}$ and the 


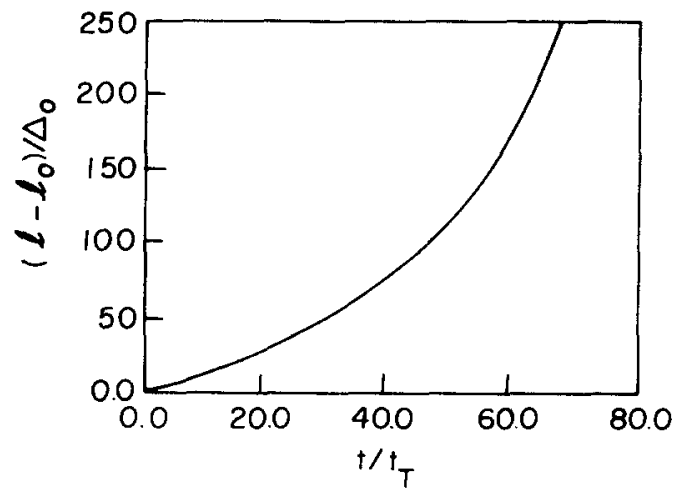

(a)

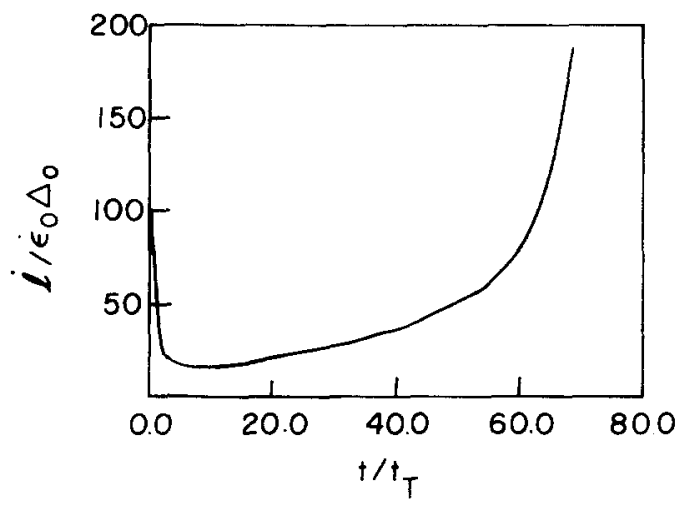

(b)

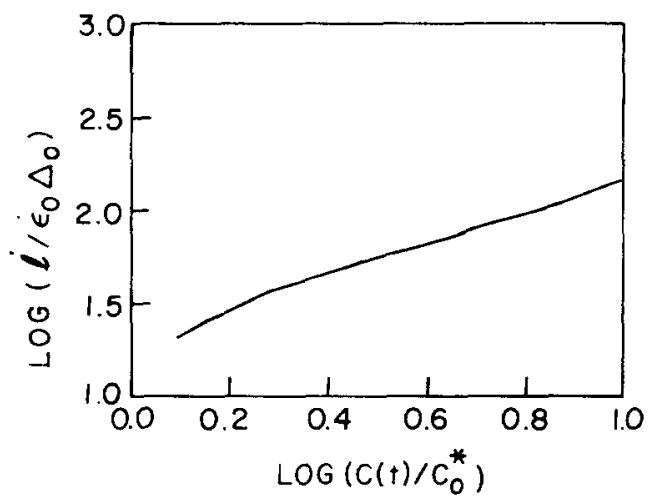

(c)

Fig. 12. Low load case, $(\bar{\sigma} / E)_{t=0}=0.025$. (a) Crack growth versus time since load application. (b) Crack growth rate, $l$, versus elapsed time. (c) Crack growth rate, $\dot{l}$, versus $C(t)$.

$C$-integral is significant. The value of the apparent critical failure strain in Fig. $11 \mathrm{~b}$ is only slightly larger than the value in Fig. $10 \mathrm{~b}$.

This difference in apparent critical failure strain between Figs. 9b, $10 \mathrm{~b}$ and $11 \mathrm{~b}$ may be due to the decreased role of diffusion at higher stress levels. In the constitutive model used in the calculations, grain boundary void growth occurs by the combined process of grain boundary diffusion and dislocation creep in the adjacent grains, see (2.13) to (2.15). Now, the creep strain rate increases with stress like $\sigma^{n}$, where here $n=5$. The crack speed, $i$, also increases with increasing stress level and, for a power law creeping material under extensive creep conditions, $i \propto\left(C^{*}\right)^{n /(n+1)} \propto \sigma^{n}$. Since the time for creep strain accumulation is inversely proportional to the crack speed, the apparent critical failure strain varies as $\sigma^{n} / i$. Hence, for $C^{*}$ controlled crack tip fields and for creep strain controlled grain boundary void growth, a constant apparent failure strain is expected. However, at lower stress levels, diffusion plays a more important role and, since the grain boundary diffusion rate depends linearly on stress, this destroys the scaling. If at low stress levels the crack velocity does not increase as rapidly as stress to the fifth power, the ratio $\sigma^{n} / i$ increases with stress, and the apparent failure strain would also increase with stress. This scenario is consistent with the behavior seen in Figs. 9b, $10 \mathrm{~b}$ and $11 \mathrm{~b}$. 


\subsection{Crack growth rates}

The crack growth rates obtained from calculations using the meshes in Figs. 3 and 4 are pieced together in Figs. 12, 13 and 14. In all three cases, the crack begins growth at a high rate and then the growth rate slows rapidly because of crack tip stress relaxation due to creep. For the mesh in Fig. 3, where the crack tip is represented by a semi-circular notch, the crack grows straight ahead. However, for the mesh in Fig. 4, where the crack tip is a jump in boundary condition, the crack grows off the initial crack line by one row of elements and then grows parallel to the initial crack line. This tendency for creep damage accumulation to occur directly ahead for rounded notches and at an angle for sharp cracks is consistent with previous theoretical and experimental results, $[11,12]$, and [47].

For the low load case shown in Fig. $12, i$ reaches a minimum at $t \approx 10 t_{T}$. At this point the crack is long enough for further lengthening of the crack to cause the intensity of the near-tip stresses to increase. Hereafter, the crack growth rate increases with time. The crack growth rate histories for the high load and the ultra-high load cases are similar. The upturn in the crack growth rate occurs at a shorter time because the crack length is increasing at a faster rate so that the effect of a longer crack is reached sooner. It may be noted that

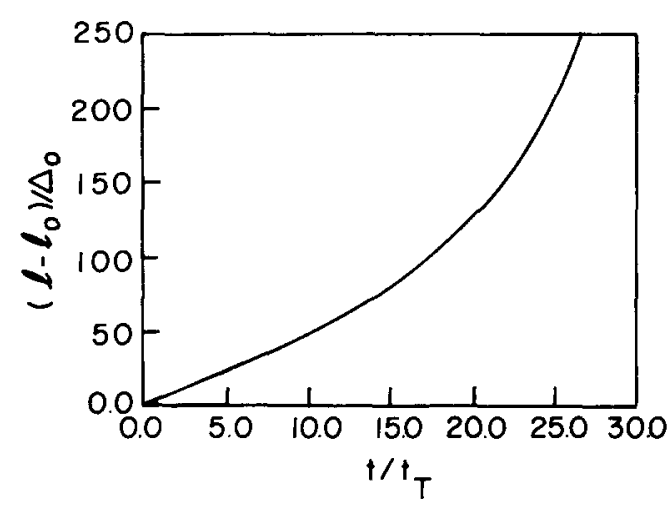

(a)

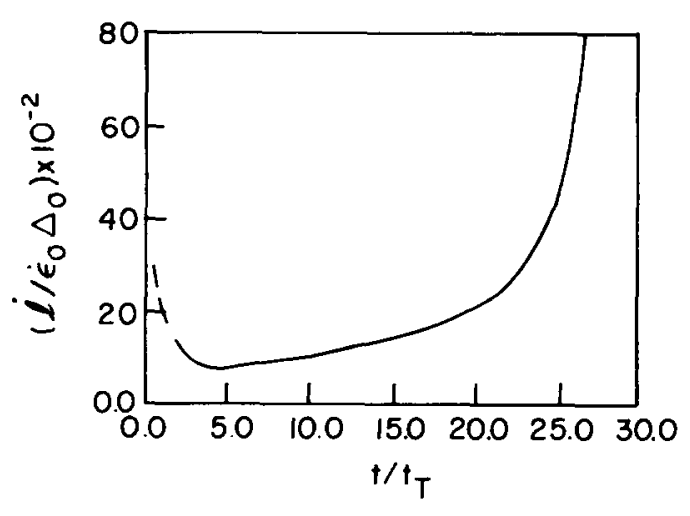

(b)

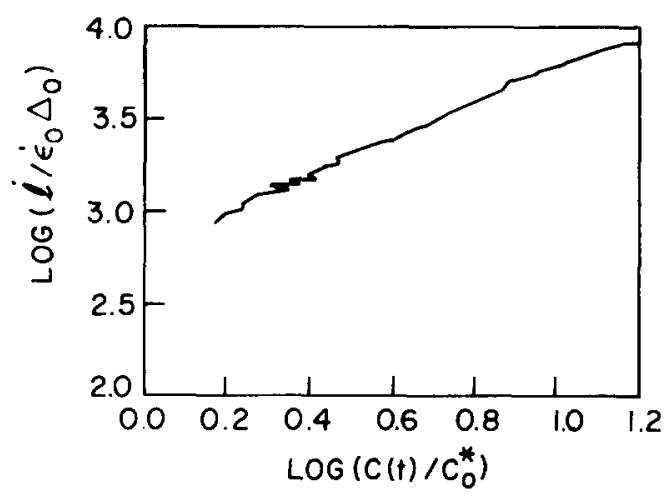

(c)

Fig. 13. High load case, $(\bar{\sigma} / E)_{t=0}=0.05$. (a) Crack growth versus time since load application. (b) Crack growth rate, $l$, versus elapsed time. (c) Crack growth rate, $\dot{l}$, versus $C(t)$. 


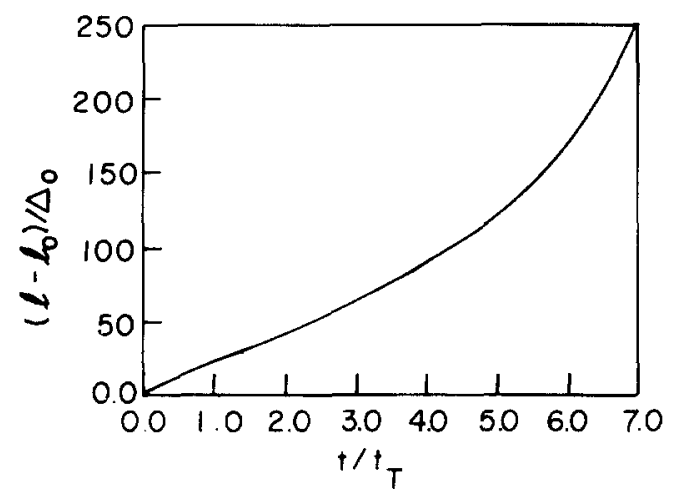

(a)

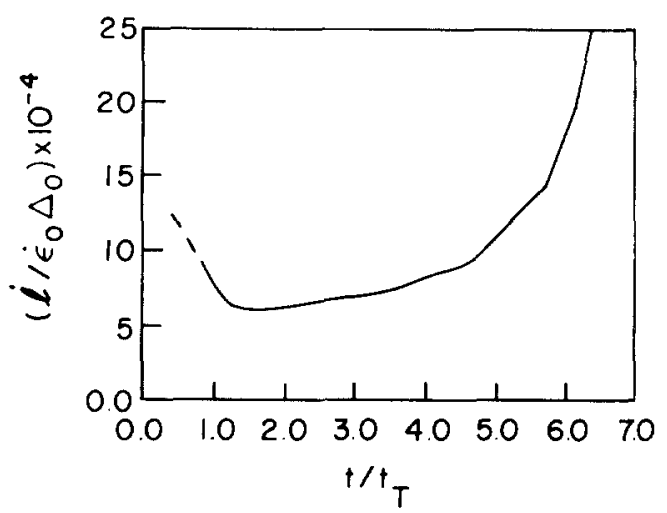

(b)

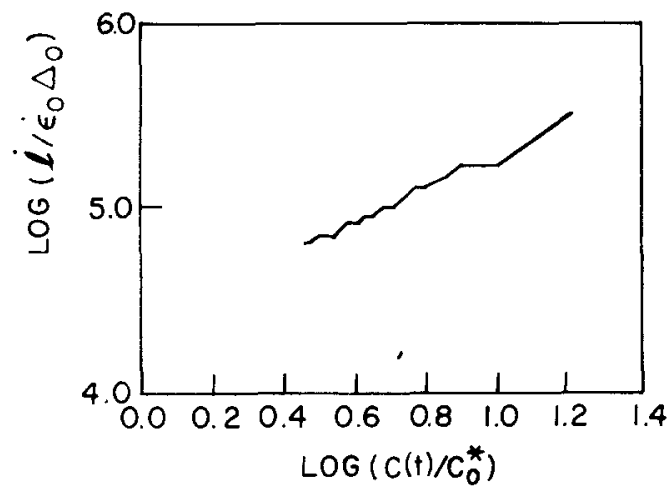

(c)

Fig. 14. Ultra-high load case, $(\bar{\sigma} / E)_{t=0}=0.1$. (a) Crack growth versus time since load application. (b) Crack growth rate, $i$, versus elapsed time. (c) Crack growth rate, $i$, versus $C(t)$.

the growth rates in Fig. 13 are about two orders of magnitude larger than those in Fig. 12 while the growth rates in Fig. 14 are about four orders of magnitude larger.

The logarithm of the crack growth rate is plotted as a function of $\log \left(C(t) / C_{0}^{*}\right)$ in Figs. $12 \mathrm{c}$, $13 \mathrm{c}$ and $14 \mathrm{c}$. These three separate plots are combined in Fig. 15, where each segment is normalized by the value of $C_{0}^{*}$ for the case in Fig. 12c. We have also included Saxena's $C_{t}$ parameter [34] in the figure. Over the entire range in this figure, the creep crack growth rates are well approximated by a power law expression, i.e. $i \propto C(t)^{m}$ or $i \propto C_{t}^{m}$ with $m=0.88$. In other words, all three sets of crack growth rates for the compact specimen are fairly well correlated by the $C$-integral or by $C_{t}$.

\subsection{Crack growth for a relatively brittle material}

Previous calculations have been based on the failure criterion of cavity coalescence at $a / b=1$. For relatively brittle materials it is plausible that the link-up process will occur at a lower value of $a / b$. In order to explore the behavior of a more brittle material response, a calculation was carried out for a material with the same properties as in the previous cases except that the value of the grain boundary diffusion parameter is $L / a_{0}=400$ and $a / b=0.2$ is used as the failure 


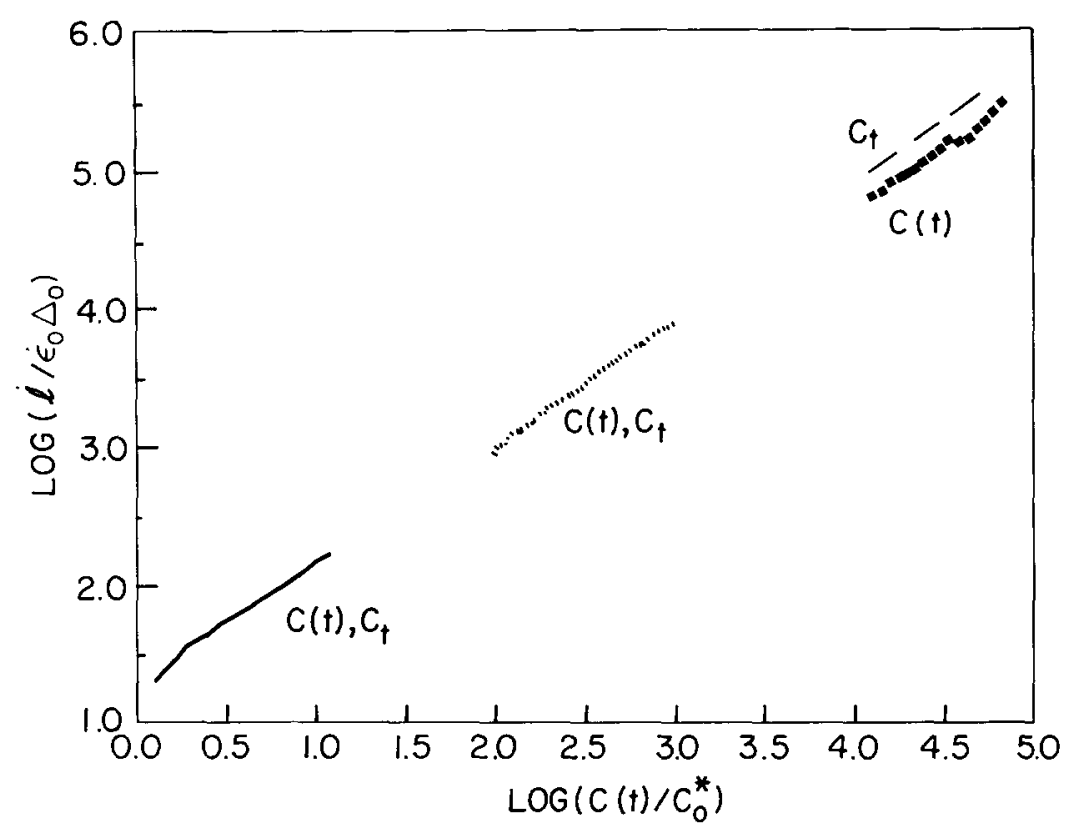

Fig. 15. Log of the crack growth rate, $l$, plotted against the $\log$ of $C(t)$, for the complete range of crack growth rates. Data for the low, high and ultra high load cases are given by the three distinct segments. The growth rates versus $C_{t}$ are also indicated.

criterion instead of $a / b=1$. The high load case, $(\bar{\sigma} / E)_{t=0}=0.05$ or $\left(K_{\mathrm{l}} / \sigma_{0}\right)^{2} / \Delta_{0}=1250$, is analyzed. For this load case, a calculation was also carried out using $L / a_{0}=400$, and a failure criterion $a=b$. The results of this calculation do not differ in any significant way from the high load case results obtained previously for $L / a_{0}=40$.

For the material characterized by brittle response, the calculated crack growth rates are nearly five hundred times faster than the growth rates for more ductile material characterized by $L / a_{0}=40$ and $a / b=1$ and subject to the same applied load. Over a very short period, $t / t_{T}<0.01$, the crack has extended more than 25 percent of the ligament specimen. The stress fields determined by the finite element calculations at different times are given in Fig. 16. At the onset of growth, the creep zone extends less than $20 R_{0}$ ahead of the crack tip, so that the tip is surrounded by essentially elastic material. During early growth, $\Delta l<700 R_{0}$, the calculated stresses are much higher than those of the HRR or HR singularities but agree well with the $K$-field. For larger amounts of crack growth the near-tip stress fields gradually shift away from the elastic $K$ fields.

Figure 17 shows the crack growth and the growth rates for the brittle material behavior. The crack begins growth at a high rate and the growth rate increases for a short time. The growth rate reaches a maximum at $t / t_{T}<3 \times 10^{-4}$, and then it decreases. This behavior is completely different from the growth rate history for the more ductile material as shown in Figs. 12b, 13b and 14b.

\section{Discussion}

We begin by considering the behavior of the more ductile material. In the low load case, a sizable creep zone has been established by the time crack growth initiates. The maximum radial 


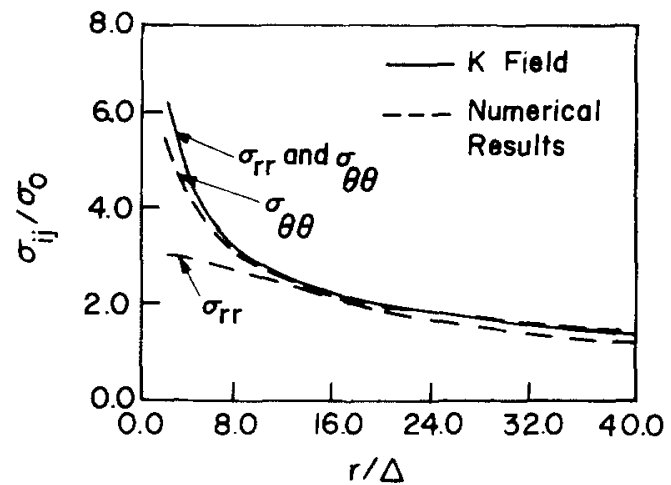

(o)

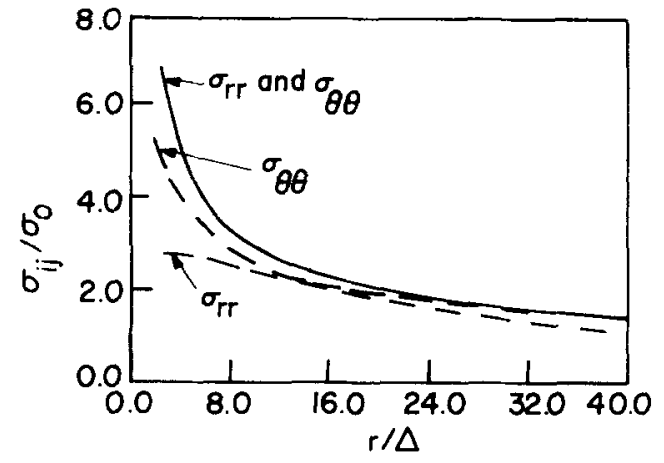

(b)

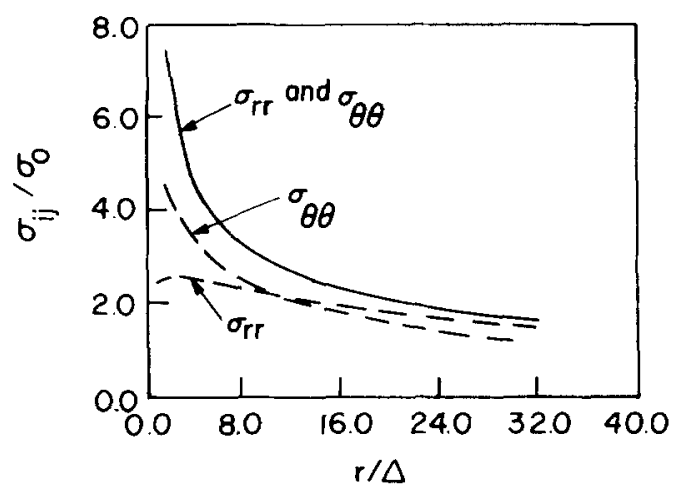

(c)

Fig. I6. Response of brittle material to high load case, $(\bar{\sigma} / E)_{t=0}=0.1$. Comparison of computed stress fields with the linear elastic singular fields at $t / t_{T}=0.361 \times 10^{-3}, 0.121 \times 10^{-2}, 0.268 \times 10^{-2}$, and for crack growths, $\left(l-l_{0}\right) /\left(W-l_{0}\right)=0.025,0.077,0.145$ respectively. The mesh in Fig. 4 is used for the calculation.

extent of the creep zone is about $600 R_{0}$ (or $30 \Delta_{0}$, where $\Delta_{0}$ is the initial notch opening in Fig. 3), though the distance directly ahead of the crack tip is considerably smaller, about $90 R_{0}$ (or $4.5 \Delta_{0}$ ). The crack growth rate is highest at the instant growth begins. Then, it decreases due to the near-tip fields being dominated by stress relaxation. In time, when the crack has grown by a sufficient amount for the longer crack to cause $C(t)$ to increase, the crack speed, $i$, also increases. Indeed, the history of the crack growth rates in Fig. 12b correlates fairly well with the trend of $C(t)$, Fig. 9a. Figures 5 and 6 show that the HRR-type field has a finite region of dominance so that we can make a case for $C(t)$ as the parameter for correlating growth rates for this load history.

For the high load case at the initiation of growth, the size of the creep zone ahead of the crack tip is about $40 R_{0}$ (or $2 \Delta_{0}$ ). For a short time after initiation the crack growth rate, $\dot{l}$, is greater than the rate of expansion of the creep zone, $\dot{r}_{c}$, so that growth effects dominate. Figure 7a shows that, after an amount of growth corresponding to 0.1 percent of the ligament or $25 R_{0}$, the HR field is well established and is embedded within an HRR-type field that dominates at distances beyond $10 \Delta$. The crack growth rate decreases with time and near the transition time, $i<\dot{r}_{c}$. At this point and beyond, creep strain rates dominate so that the near-tip fields are of the HRR-type (see Fig. 7b). During this period, the crack speed slows down even more. For $t>4 t_{T}$, the crack has grown enough for $C(t)$ to increase due to the effect of the longer crack. In this case, 


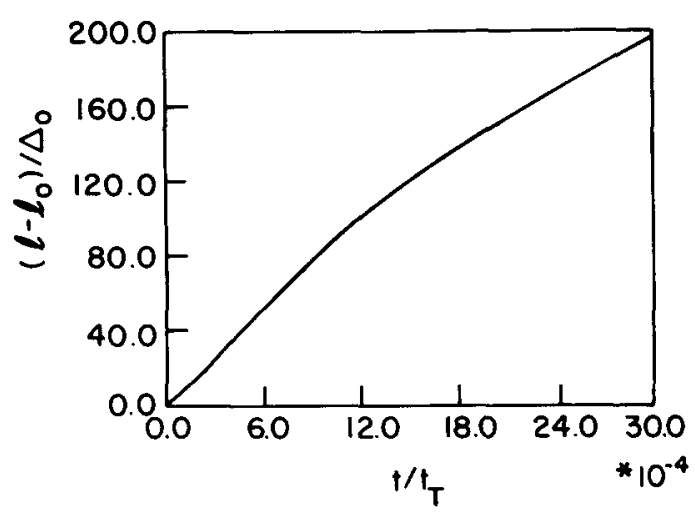

(a)

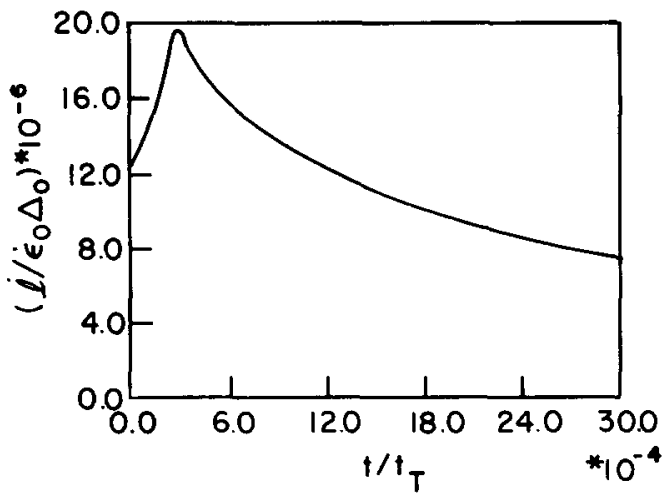

(b)

Fig. 17. Response of brittle material to high load case, $(\bar{\sigma} / E)_{t=0}=0.1$. (a) Crack growth versus time since load application. (b) Crack growth rate, $l$, versus elapsed time.

the history of the growth rates is similar to that for the low load case, although the growth rate is two orders of magnitude higher (compare Figs. 12 and 13).

For the ultra-high load case, the creep zone extends less than $20 R_{0}$ ahead of the crack tip at the onset of crack growth. For a short time, $i>\dot{r}_{c}$, so that the crack tip is surrounded by an essentially elastic region dominated by the elastic $K$ field (see Fig. 8a). As time progresses, the crack growth rate slows down sufficiently so that $i<\dot{r}_{c}$. Hereafter, the crack tip is surrounded by a sizable creep zone and fields of the HRR-type dominate (see Fig. $8 \mathrm{~b}$ ). The crack growth rate history is similar to those for the lower load levels - however the crack growth rate is about four orders of magnitude higher than those for the low load case (compare Figs. 12 and 14).

The brittle material presents a growth history which stands in marked contrast to the history described above. At initiation, the creep zone extends a distance less than $20 R_{0}$ beyond the crack tip. The growth rate starts out high and increases even further, reaching a maximum at $t / t_{T}=3 \times 10^{-4}$, as shown in Fig. $17 \mathrm{~b}$. At this time, the crack tip is practically ahead of the creep zone so that the cavity growth rate cannot develop fast enough to maintain the high rate of growth. (It may be recalled that the cavity growth rate is enhanced by creep straining). At this stage and beyond, the crack growth rate decreases. Nevertheless, the lower growth rates are still several orders of magnitude higher than those for the more ductile material.

To illustrate this, Fig. $18 \mathrm{~b}$ shows the creep zone in the brittle material at $t / t_{T} \approx 10^{-3}$, at which time the crack has grown an amount equal to 6.5 percent of the ligament. The initial position of the crack tip is indicated. It can be seen that the tip is surrounded by a small creep zone at the onset of growth. During a very short time, $0<t / t_{T}<4 \times 10^{-4}$, the growth rate increases rapidly so that the tip runs ahead of the creep zone. Thus, the crack advances through elastic material, as indicated by the absence of the creep zone along a portion of the crack path. After this initial spurt of growth, the growth rate decreases and a small creep zone forms ahead of the crack tip as indicated by the reappearance of a second creep zone. In contrast, for the ductile material, the crack is always growing into highly strained material. Figure 18a shows the contours of constant creep strain in the ductile material at a comparable stage of crack growth (the difference in scale should be noted).

The stress relaxation due to constrained creep and the stress increase associated with the instantaneous elastic response to crack growth are competing effects on the near-tip fields. For 


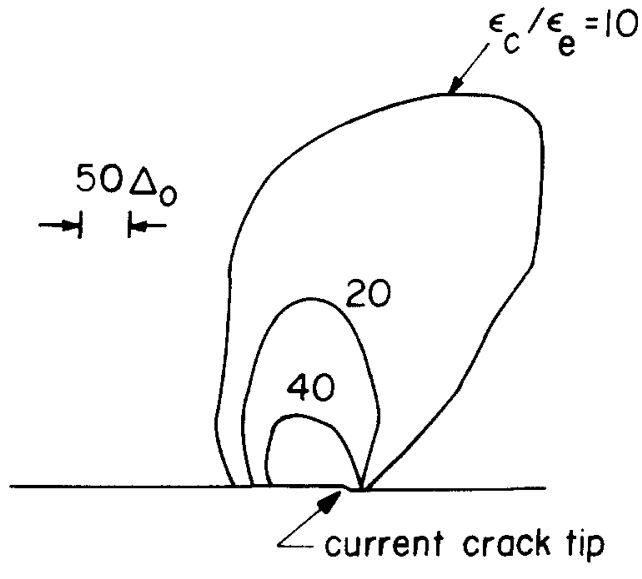

(a)

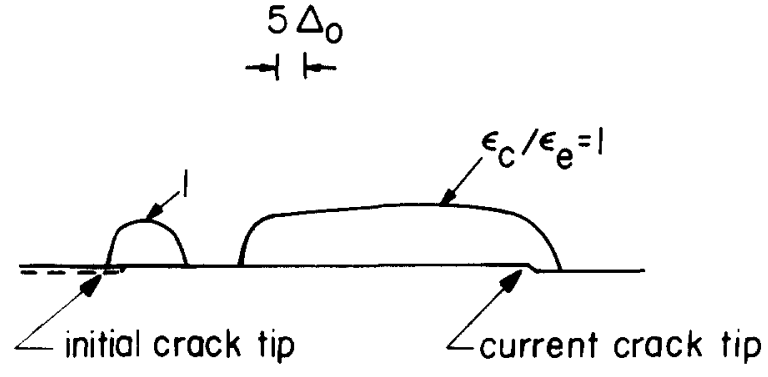

(b)

Fig. 18. Comparison of creep zones at comparable amounts of crack growth. (a) Ductile material at $t / t_{T}=40.2$ for $\mathrm{crack}$ growth, $\left(l-l_{0}\right) /\left(W-l_{0}\right)=0.0628$ for the low load case, $(\bar{\sigma} / E)_{t=0}=0.025$. (b) Brittle material at $t, t_{T}=0.979 \times 10^{-3}$ for crack growth, $\left(l-I_{0}\right)\left(W-l_{0}\right)=0.0652$ for the high load case, $(\bar{\sigma} / E)_{t=0}=0.05$.

the ductile material and for the crack geometry considered in this study, our calculations show that strong effects from crack growth are confined to the early growth history. Even at the very high loads which lead to high growth rates, growth effects are greatly diminished for times approaching the transition time. The stress fields plotted in Figs. 5 through 8 for the low, high and ultra-high load cases show that over the greatest range of times and including the transient creep regime, creep strain rates dominate and the near-tip fields are of the HRR-type. Thus, it is not surprising that for growth rates that range over five orders of magnitude, $i$ scales linearly with $C(t)^{n /(n+1)}$ or $C_{t}^{n /(n+1)}$ as shown in Fig. 15. In a previous study, which was confined to small scale creep [13], we obtained growth histories and correlations of growth rates with $C(t)$ which are quite similar to those found here. The extent to which our results depend on the values of material parameters employed and on the crack geometry analyzed remains to be determined. However, there is reason to believe that our results have some general validity. Riedel, Bassani, Kubo, Hui and others have pointed out that under strain controlled growth, the growth rate in a nonlinearly viscous material should be proportional to $\left(C^{*}\right)^{n /(1+n)}$. The latter result can be extended to an elastic nonlinear viscous material in which case $i \propto C(t)^{n / 1+n)}$ (see the discussion in $[1,2])$. For $n=5$, the exponent $n /(1+n)$ equals 0.83 and this value agrees well with our calculated slope of 0.88 in Fig. 15.

Using a one-dimensional damage model, Wu et al. [41] and Bassani et al. [42] have investigated a full range of crack growth histories under small scale creep. In the growth regimes where the HR field dominates the near-tip response, their analysis predicts rather complex crack growth histories and they make the point that neither $K$ nor $C(t)$ uniquely correlates $i$ under small scale creep conditions. Indeed, it was because of such theoretical and similar experimental results, as well as the fact that $C(t)$ cannot be determined from measurements, that Saxena proposed $C_{t}$ as an alternative crack tip characterizing parameter $[3,34]$. As discussed by Bassani et al. $[35,36], C_{t}$ is proportional to the rate of expansion of the creep zone, $\dot{r}_{c}$. If the rate of damage accumulation is proportional to $\dot{r}_{c}$, then a case can be made for $i$ to correlate with $C_{t}$. It is worth pointing out that Riedel [40] has observed that the HR singularity has no range of validity in a Kachanov type damage mechanics model and therefore the complex growth histories reported in $[41,42]$ are not found in analyses based on such 
damage mechanics models. The subject of crack growth under small scale creep requires further study.

In our studies, we did not address the subject of primary creep although there are many engineering materials where the strains due to primary creep are comparable to secondary creep. Primary creep effects on overall deformation rates and crack growth rates have been addressed by Riedel and Detampel [51] and Leung, McDowell and Saxena [52]. The former investigators demonstrated that the $C_{t}$ parameter correlates crack growth rates during the transient period when primary creep dominates the response of the specimen. At later times secondary creep dominates and $C_{t}$ and $C^{*}$ are equivalent parameters.

In summary, there is sufficient evidence that within the small scale damage approximation, a practical range of conditions exists under which crack growth rates can be predicted by $C(t)$ as well as by $C_{t}$. Therefore, it seems appropriate to provide an engineering estimate of $C_{t}$. We designate the steady state value of $C$-integral based on the current crack length by $C^{*}$ and for some geometries the value can be found in a handbook by Kumar et al. [33]. For a growing crack, Bassani et al.'s [35] interpolation relation (3.11) is modified to become

$$
\bar{C}_{t}=\left[\alpha\left(\frac{t_{T}^{*}}{t}\right)^{(n-3) /(n-1)}+1\right] C^{*},
$$

where $t_{T}^{*}$ is the transition time based on the current crack length and therefore accounts for a changing crack length. For the slowly growing crack which is the case of engineering interest, the behavior of $C^{*} / C_{0}^{*}$ is shown in Fig. 19. It can be seen that for times larger than about $60 t_{T}$, $C_{0}^{*}$, which is based on the initial crack length, underestimates the actual value, $C^{*}$, by a considerable amount. Using the current crack length to calculate $C_{t}$ in (3.12) does give a fair

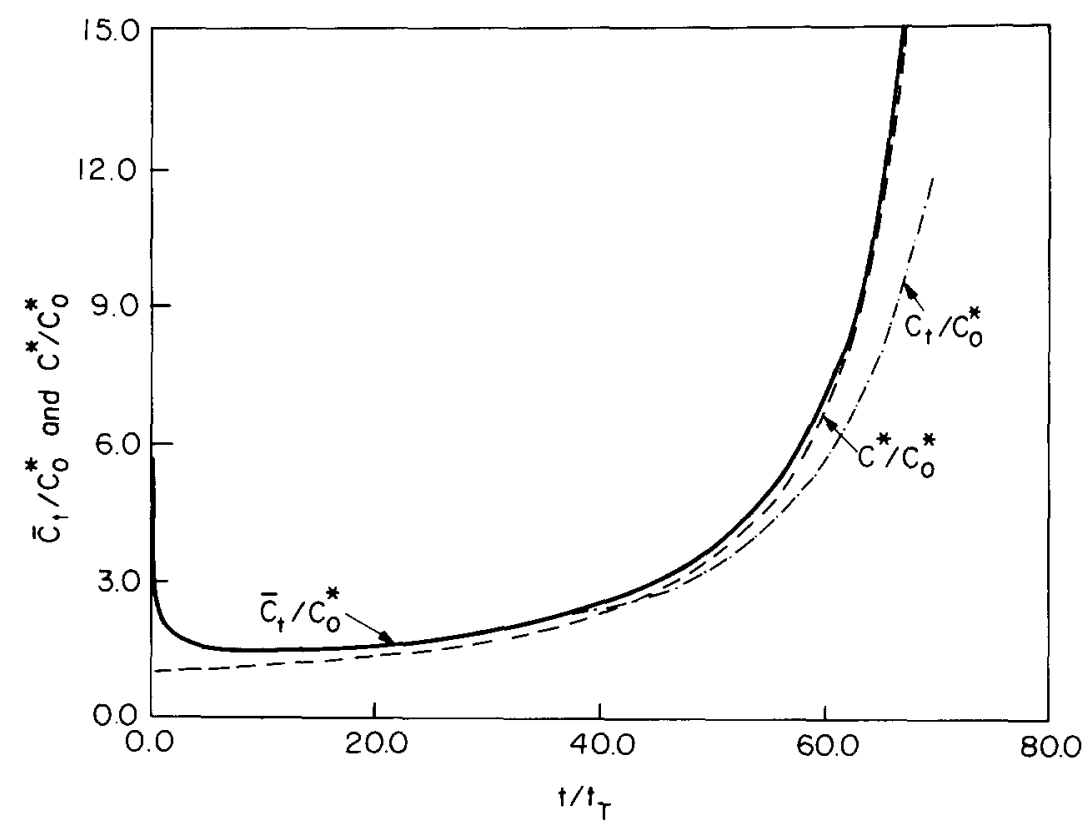

Fig. 19. Comparison of $C_{t}, \bar{C}_{t}$ and $C^{*}$ for a growing crack in a compact specimen for the low load case, $(\bar{\sigma} / E)_{t=0}=0.025$. 
estimate of $C^{*}$. As is also shown in Fig. 19, an even better estimate of the actual value at long times is $\bar{C}_{t}$ determined by (5.1). At short times $\bar{C}_{t}$ also captures stress relaxation effects, behaving nearly like $C(t)$, even though for times less than the transition time $C_{t}$ (and $\bar{C}_{t}$ ) have a dependence on time different from that of $C(t)$.

The theoretical basis and the supporting experimental data are there for us to conclude that $C(t)$ is the relevant load parameter when creep strain rates dominate the near tip response. This conclusion is no longer tenable at high crack growth rates where creep and elastic strain rates are comparable. In the sense that $\Phi$ in the definition of $C(t)$ in (3.3) is based on the expression for a pure power law creeping solid, $C(t)$ is not defined at high growth rates since elastic strain rates are then important at the crack tip. In contrast, $C_{t}$ is still related to the rate of expansion of the creep zone $\dot{r}_{c}$ in the highly transient regime, and to the extent that $\dot{r}_{c}$ is driven primarily by the extending crack, Saxena has suggested that $C_{t}$ remains a viable parameter for correlating $i[53]$. The idea merits investigation.

In the present study, damage has been largely confined to the region near the tip. When damage is rather diffuse, a case cannot be made for a fracture mechanics approach based on $C(t), C_{t}$ and $C^{*}$. The competition between the diffuse damage mode of failure and the growth of a single dominant crack has been discussed by Hsia, Argon and Parks [54].

\section{Acknowledgments}

The support of the Materials Research Group at Brown University, funded by the National Science Foundation through Grant DMR-8714665 is gratefully acknowledged. The computations reported on here were carried out on an Alliant FX-8 computer at the Brown University, Division of Engineering, Computational Mechanics Facility. Helpful discussions with Dr. F.Z. Li are gratefully acknowledged.

\section{References}

1. H. Riedel, Fracture at High Temperatures, Springer-Verlag Berlin, Heidelberg (1987).

2. H. Riedel, in Advances in Fracture Research, Proceedings of ICF7, 2 (1989) 1495-1523.

3. A. Saxena. in Fracture Mechanics: Microstructure and Micromechanisms, American Society of Metals (1989) $283-334$.

4. J.W. Hutchinson, Journal of Applied Mechanics 50 (1983) 1042-1051.

5. D.R. Hayhurst, P.R. Brown and C.J. Morrison, Philosophical Transactions of the Royal Society of London A311 (1984) 131-158.

6. D.R. Hayhurst, F.A. Leckie, in Mechanical Behavior of Materials, Proceedings of ICM4, J. Carlsson and N.G. Ohlson (eds.), Vol. 2 (1984) 1195-1212.

7. L.M. Kachanov, The Theory of Creep, English translation edited by A.J. Kennedy, Boston Spa, Wetherby (1960).

8. J.W. Hutchinson, Acta Metallurgica 31 (1983) 1079-1088

9. V. Tvergaard, Acta Metallurgica 32 (1984) 1990-1997.

10. V. Tvergaard, Journal of the Mechanics and Physics of Solids 32 (1984) 373-393.

11. V. Tvergaard, Mechanics of Materials 4 (1985) 181-196.

12. V. Tvergaard, International Journal of Fracture 31 (1986) 183-209.

13. F.Z. Li. A. Needleman and C.F. Shih, International Journal of Fracture 38 (1988) 241-273.

14. A. Needleman, in Plasticity of Metals at Finite Strain: Theory, Computation and Experiment, E.H. Lee and R.L. Mallett (eds.), Stanford University, Stanford, CA (1982) $387-436$.

15. B.F. Dyson, Metal Science 10 (1976) 349-353.

16. J.R. Rice, Acta Metallurgica 29 (1981) 675-681. 
17. A. Needleman and J.R. Rice, Acta Metallurgica 28 (1980) 1312-1315.

18. T.-L. Sham and A. Needleman, Acta Metallurgica 31 (1983) 919-926.

19. D. Peirce, C.F. Shih and A. Needleman, Computers and Structures 18 (1984) 875-878.

20. V. Tvergaard, Journal of the Mechanics and Physics of Solids 30 (1982) 399-425.

21. J.W. Hutchinson, Acta Metallurgica 31 (1983) 1079-1088.

22. J.W. Hutchinson, Journal of the Mechanics and Physics of Solids 16 (1968) 13-31.

23. J.R. Rice and G.F. Rosengren, Journal of the Mechanics and Physics of Solids 16 (1968) 1-12.

24. J.L. Bassani and F.A. McClintock, International Journal of Solids and Structures 17 (1981) 467-476.

25. H. Riedel and J.R. Rice, in Fracture Mechanics: Twelfth Conference, STP 700, American Society for Testing and Materials, Philadelphia (1980) 112-130.

26. K. Ohji, K. Ogura and S. Kubo, Journal of the Society of Material Science (Japan) 29 (1980) 465-471 (in Japanese).

27. R. Ehlers and H. Riedel, in Advances in Fracture Research, D. Francois (ed.), ICF-5, Cannes, France (1981) 691-698.

28. F.Z. Li, A. Needleman and C.F. Shih, International Journal of Fracture 36 (1988) 163-186.

29. J.R. Rice, Journal of Applied Mechanics 35 (1968) 379-386.

30. K. Ohji, K. Ogura and S. Kubo, Preprint of Japanese Society of Mechanical Engineers, No. 640-11 (1974) 207 (in Japanese).

31. J.D. Landes and J.A. Begley, in Mechanics of Crack Growth, STP 590, American Society for Testing and Materials, Philadelphia (1976) 128-148.

32. K.M. Nikbin, G.A. Webster and C.E. Turner, in Cracks and Fracture, STP 601, American Society for Testing and Materials, Philadelphia (1976) 47-62.

33. V. Kumar, M.D. German and C.F. Shih, An engineering approach for elastic-plastic fracture analysis. EPRI NP-1931, Electric Power Research Institute, Palo Alto, California (1981).

34. A. Saxena, in Fracture Mechanics: Seventeenth Volume, STP 905, American Society for Testing and Materials, Philadelphia (1986) 185-201.

35. J.L. Bassani, D.E. Hawk and A. Saxena, in Nonlinear Fracture Mechanics: Vol. 1 - Time-Dependent Fracture, ASTM STP 995 (1989) 7-26.

36. J.L. Bassani and C.S. Liu, The correlation of transient crack growth under small scale creep conditions. To be published.

37. C.Y. Hui and H. Riedel, International Journal of Fracture 17 (1981) 409-425.

38. C.Y. Hui, in Elastic-Plastic Fracture: Vol. 1 - Inelastic Crack Analysis, ASTM STP 803 (1983) 573-593.

39. H. Riedel and W. Wagner, in Advances in Fracture Research, D. Francois (ed.), ICF-5, Cannes, France (1981) 683-690.

40. H. Riedel, International Journal of Fracture 42 (1990) 173-188.

41. F.H. Wu, J.L. Bassani and V. Vitek, Journal of the Mechanics and Physics of Solids 34 (1986) 455-475.

42. J.L. Bassani, D.E. Hawk and F.H. Wu, in Nonlinear Fracture Mechanics: Vol. 1 - Time-Dependent Fracture, ASTM STP 995 (1989) 68-95.

43. C.Y. Hui and V. Banthia, International Journal of Fracture 25 (1984) 53-67.

44. C.Y. Hui and K.C. Wu, International Journal Fracture 31 (1986) 3-16.

45. R.A. Ainsworth and P.J. Budden, Crack tip fields under on-steady creep conditions ii: estimates of associated crack growth. CEGB Report RD/B/6006/R88 (1988).

46. K. Watanabe, S. Tani, S. Yoshioka and Y. Yabe, International Journal of Fracture 39 (1989) 287-300.

47. K.J. Hsia, A.S. Argon and D.M. Parks, Mechanics of Materials, 11 (1991) 19-42.

48. H. Tada, P.C. Paris and G.R. Irwin, The Stress Analysis of Cracks Handbook, Del Research Corporation, Hellertown, PA (1973).

49. J.D. Eshelby, in Progress in Solid State Physics, Vol. 3, F. Seitz and D. Turnbull (eds.), Academic Press, New York (1956) 79-144.

50. F.Z. Li, C.F. Shih and A. Needleman, Engineering Fracture Mechanics 21 (1985) 405-421.

51. H. Riedel and V. Detampel, International Journal of Fracture 33 (1987) 239-262.

52. C.-P. Leung, D.L. McDowell and A. Saxena, International Journal of Fracture 36 (1988) $275-289$.

53. A. Saxena, private communication (1990).

54. K.J. Hsia, A.S. Argon and D.M. Parks, in Proceedings of the International Conference on Creep in Engineering Materials and Structures, to be published.

55. V. Tvergaard, Acta Metallurgica 35 (1987) 923-933.

56. D.E. Hawk and J.L. Bassani, Journal of the Mechanics and Physics of Solids 34 (1986) 191-212. 